Recepción: 22/03/2019

Aceptación: 23/04/2019

Publicación: 05/05/2019

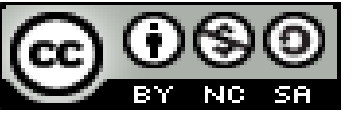

Ciencias de la salud

Artículo de investigación

\title{
Calidad del servicio de enfermería en la Fundación Cottolengo: Análisis con instrumento "Servqhos-E"
}

\section{Quality of the nursing service at the Cottolengo Foundation: Analysis with instrument "Servqhos-E"}

\section{Qualidade do serviço de enfermagem da Fundação Cottolengo: Análise com instrumento "Servqhos-E"}

Cristhian Geovanny Marín-Tello ${ }^{\text {I }}$

cristhianoamlt@gmail.com

Leiver Henry Rivera-Chávez II

leiver.river@gmail.com

Pedro Luís Fernández-Sánchez ${ }^{\text {III }}$

pedrofernandez84@hotmail.com
Nancy Monserrate Macías-Palacios IV

dranancymacias@yahoo.es

Jorge Alberto Cañarte-Alcívar ${ }^{\mathrm{V}}$

.jcanarte@utm.edu.ec

Correspondencia: cristhianoamlt@gmail.com

I Magíster en Gerencia de la Salud, Doctor en Medicina y Cirugía, Director Médico Fundación Cottolengo. Manta. Ecuador.

${ }^{\text {II }}$ Médico Cirujano, Medico General en Funciones Hospitalaria Hospital General IESS Manta. Ecuador.

III Médico Cirujano, Medico General en Funciones Hospitalaria - Hospital General Dr. Napoleón Dávila Córdova. Chone. Ecuador.

${ }^{\text {IV }}$ Médico Cirujano, Medico General en Funciones Hospitalaria Hospital General IESS, Manta. Ecuador.

${ }^{\vee}$ Máster en Investigación Clínica y Epidemiológica Doctor en Medicina y Cirugía, Doctor en Salud Pública, Docente Investigador, Universidad Técnica de Manabí, Manta, Ecuador. 


\section{Resumen}

El propósito de este estudio es analizar la calidad del Servicio de Enfermería a través de la aplicación de un instrumento de investigación que mide la percepción de la calidad del servicio, que a su vez es valorado por nuestros pacientes, que están hospitalizados en la Fundación Cottolengo de la Ciudad de Manta. La metodología aplicada parte de un enfoque cuantitativo y cualitativo, para ello se aplicó un instrumento adaptado a la Calidad del Servicio de Enfermería. El instrumento fue aplicado a 65 pacientes que se ingresaron al área de hospitalización en nuestra fundación. La validación del instrumento mediante el Alfa de Cronbach fue de 0.887 por lo tanto es altamente adaptado a la realidad. Se encontró un nivel de Satisfacción Global de 86.15\% y un porcentaje de Recomendación de la Fundación del 96.92\%, lo cual, según la teoría de desconfirmación de expectativas de Oliver, indica altos niveles de calidad percibida en la Fundación. Es hasta el momento el primer estudio en el Ecuador que se realiza utilizando el instrumento, SERVQHOS-E para medir Calidad del Servicio de Enfermería después de haber sido validado en el vecino país del norte, lo cual obligó al investigador a crear una base de datos para el procesamiento de la información, la cual se elaboró en el programa SPSS versión 24. De acuerdo a los resultados obtenidos se realizará un plan de capacitación continua para el personal de enfermería de acuerdo a su cargo.

Palabras Claves: Calidad de servicio; enfermería; cuidados paliativos; Fundación Cottolengo.

\section{Abstract}

The purpose of this study is to analyze the quality of the Nursing Service through the application of a research instrument that perceives the quality of the service, which in turn is valued by our patients, who are hospitalized in the Cottolengo Foundation of the City of Manta. The applied methodology starts from a quantitative and qualitative approach, for which an instrument adapted to the Quality of the Nursing Service was applied. The instrument was applied to 65 patients who were admitted to the hospitalization area in our foundation. The validation of the instrument using Cronbach's alpha was 0.887 and therefore highly adapted to reality. A Global Satisfaction level of $86.15 \%$ and a Foundation Recommendation rate of $96.92 \%$ was found, which, according to Oliver's theory of Oliver's lack of expectation, indicates high levels of quality perceived in the Foundation. 
It is the first study in Ecuador, which is performed using the instrument, SERVQHOS-E to measure Quality of Nursing Service after having been validated in the neighboring country of the north, which forced the researcher to create a base of data for the processing of the information, which was elaborated in the program SPSS version 24. According to the results obtained, a continuous training plan will be carried out for the nursing staff according to their position.

Keywords: Quality of service; nursing; palliative care; Cottolengo Foundation.

\section{Resumo}

O objetivo deste estudo é analisar a qualidade do Serviço de Enfermagem por meio da aplicação de um instrumento de pesquisa que mensura a percepção da qualidade do serviço, que por sua vez é valorizado por nossos pacientes, que são internados na Fundação Cottolengo da cidade de Manta. A metodologia aplicada baseia-se em uma abordagem quantitativa e qualitativa, para a qual foi aplicado um instrumento adaptado à Qualidade do Serviço de Enfermagem. O instrumento foi aplicado em 65 pacientes admitidos na área de internação em nossa fundação. A validação do instrumento pelo alfa de Cronbach foi de 0,887 , portanto, é altamente adaptada à realidade. Foi encontrado um nível de Satisfação Global de $86,15 \%$ e uma porcentagem de recomendação da Fundação de 96,92\%, o que, de acordo com a teoria de desconfirmação das expectativas de Oliver, indica altos níveis de qualidade percebida na Fundação. É o primeiro estudo no Equador que é feito usando o instrumento SERVQHOS-E para medir a Qualidade do Serviço de Enfermagem depois de ter sido validado no país vizinho do norte, o que forçou o pesquisador a criar um banco de dados para o processamento das informações, que foi elaborado no programa SPSS, versão 24. De acordo com os resultados obtidos, será elaborado um plano de treinamento contínuo para a equipe de enfermagem de acordo com sua posição.

Palavras-chave: Qualidade de serviço; enfermagem cuidados paliativos; Fundação Cottolengo.

\section{Introducción}

En un sentido etimológico, "calidad proviene del Latín qualitis que significa el conjunto de cualidades que constituyen la manera de ser de una persona o cosa; es un sinónimo de cualidad, clase, actitud, excelencia, categoría, casta, nobleza, superioridad entre otras muchas acepciones" 
(Galván et al. 2016). El concepto de la calidad como cultura nació en empresas de servicio al cliente, particularmente, en la industria turística, en las ramas de hotelería y de restaurantes.

En el Ecuador, la Constitución establece que los servicios de salud deben tener calidad, calidez y eficiencia, razón por la cual, es necesario establecer mecanismos que permitan valorar la satisfacción tanto del cliente interno como del cliente externo, haciendo que la calidad se convierta en un compromiso de la alta dirección, involucrando a todos los actores comprometidos en la atención en salud.

Calidad de la atención en salud se entiende; como la provisión de servicios de salud a los usuarios individuales y colectivos de manera accesible y equitativa a través de un nivel profesional óptimo, donde se tiene en cuenta el balance entre los beneficios, los riesgos y los costos, con el propósito de lograr la mayor adhesión y la mejor satisfacción de los usuarios.

Para determinar la calidad, es necesario definir mecanismos claros, precisos y ágiles, que permitan establecer procesos donde se realice la medición, la evaluación y el establecimiento de planes de mejoramiento, acordes con los hallazgos encontrados. En este contexto, la evaluación de la calidad del cuidado de enfermería es un proceso que busca alcanzar estándares de excelencia dentro de la Gerencia del Cuidado.

La satisfacción del paciente con el cuidado de enfermería ha sido reportada como el predictor más importante de la satisfacción con la hospitalización. Lo anterior es esperable, pues es el personal de enfermería el que mayor volumen representa en el hospital y el que mayor contacto tiene con los pacientes, los que esperarán que la atención de enfermería sea de calidad. La percepción de los pacientes sobre la calidad del cuidado no siempre coincide con la percepción que de éste tienen los enfermeros: los pacientes valoran altamente aspectos como el trato humanizado y el que parezcan buenos profesionales, mientras que para los enfermeros estos aspectos no tienen el mismo peso, y le dan mucha más importancia a los aspectos técnicos del cuidado.

La rotación laboral durante la noche representa factores de riesgo para desarrollar múltiples enfermedades de tipo crónico, desde los componentes biológico, psicológico y social, por lo que abordar estos riesgos permite establecer medidas que mitiguen el impacto en los trabajos nocturnos. Desde el punto de vista biológico, se ha relacionado la falta de sueño con un aumento de peso, diabetes, aumento de la presión arterial, problemas cardiacos, depresión y abuso de medicamentos. 
De igual modo, se ha dicho que el sueño hace que las hormonas del apetito se desorganicen, disminuyendo la calidad de atención del personal. Todas estas patologías y la falta de supervisión al personal de enfermería del turno nocturno, influyen en la disminución de la calidad de su servicio, por lo que se busca proponer un plan de capacitación continua.

\section{Calidad del cuidado de Enfermería}

En Colombia, han sido varias las investigaciones realizadas en torno al tema, dentro de ellas se encuentra la realizada por Ariza y Daza, quienes llevaron a cabo un estudio investigativo con el fin de determinar la calidad del cuidado de Enfermería al paciente hospitalizado en instituciones de tercer nivel en Bogotá, como resultado reportaron que en cuanto a la Estructura para la Enfermera profesional hay una Suficiencia de 63,7\% y para el personal auxiliar de $72,8 \%$, con lo cual se ubican en un nivel alto al utilizar la escala previamente establecida.

Respecto al proceso de la enfermera profesional se encontró que la Suficiencia es del 66\%, un Índice de Precisión Técnica del 66\% y una Accesibilidad Interna del 100\%; Esto significa que la enfermera profesional se encuentra en un nivel medio respecto a la Suficiencia y en un nivel regular con respecto al Índice de Precisión Técnica. Para el personal auxiliar se encontró una Suficiencia de 49,1\%, un Índice de Precisión Técnica del 46\% y una Accesibilidad del 100\%, con lo cual se concluye que la Eficiencia del Proceso está en un nivel bajo y regular respectivamente. Respecto al Impacto se encontró un grado de satisfacción de 53,3\%, lo cual indica insatisfacción por parte de los pacientes que reciben el cuidado Todo lo anterior evidencia la importancia de medir y evaluar continuamente la calidad de la atención de Enfermería desde la percepción de los usuarios que reciben la atención;

Es por eso que en Colombia, el interés por medir la calidad en las organizaciones de salud es cada día mayor, y es evidente en la medida en que muchos investigadores están validando instrumentos como el Servqual, Servqhos, Care-Q, Cucase, entre otros, con el fin de medir y evaluar de manera concreta la atención en salud y enfermería (Borré 2013).

Las limitaciones del estudio se centran en la selección de los artículos, ya que hay variadas tendencias y modelos y, por tanto, variadas escalas de evaluación de la calidad de los servicios de salud, en las que SERVQHOS y SERVQUAL son las más destacadas. El análisis realizado muestra 
que la tendencia de los estudios realizados se ubica en los años 2012 y 2014. A nivel mundial, los estudios se encuentran centralizados en España, Medio Oriente y Colombia, con una mayor aplicabilidad de la escala SERVQUAL y, en general, las investigaciones se enfocan en la evaluación de los servicios de hospitalización, hemodiálisis y atención primaria o ambulatoria. A nivel nacional, las temáticas estuvieron relacionadas con la percepción del usuario respecto a la calidad de la atención del servicio de enfermería en Boyacá y Barranquilla.

Algunos estudios presentan la escala SERVQUAL como un instrumento válido, confiable y apropiado para evaluar la calidad de los servicios desde la percepción del usuario mismo modo, algunos autores revelan que esta escala permite establecer planes de mejora en las instituciones prestadoras de servicios de salud, al ser la más empleada en el medio hospitalario para evaluar la calidad de los servicios desde la percepción de los usuarios. A diferencia de los estudios donde la escala muestra resultados favorables, dos estudios realizados en Medio Oriente refieren una valoración del servicio desfavorable desde la percepción del usuario, lo que hace necesario replantear estrategias y planes de mejora en la prestación de los servicios.

Solo un estudio realizado muestra algunos vacíos en la evaluación de las dimensiones que maneja el instrumento, generadas por las diferencias en las percepciones de los pacientes y propone una evaluación más profunda de la escala aplicada. En relación a la escala de evaluación SERVQHOS, esta ha tenido más utilidad en Colombia para evaluar la prestación de servicios del personal de enfermería mientras que en España su aplicabilidad se centra en servicios de hemodiálisis, oncología y anestesia. Cabe destacar que, a diferencia de la escala SERVQUAL, que evalúa cinco dimensiones en la calidad, la escala SERVQHOS agrupa variables relacionadas con la calidad objetiva que hace referencia a infraestructura, confort, dotación de equipos y asuntos relacionados con la calidad subjetiva a propósito del trato, comunicación e información hacia el usuario por parte del personal de salud. Se puede concluir que la satisfacción del usuario respecto a la calidad de los servicios de salud es un tema complejo relacionado con diferentes factores como edad, experiencias previas del paciente, estilo de vida, ingresos y educación.

Así mismo, en los últimos años, la percepción del paciente respecto a la calidad de los servicios se ha convertido en un factor determinante para los planes de gestión en las instituciones de salud. El análisis comparativo de la calidad de servicio del personal de enfermería del horario nocturno 
versus el diurno, se desarrolla en la fundación Cottolengo, ubicada en la ciudad de Manta, parroquia Tarqui, en la calle 295 entre avenidas 235 y 236 de la Provincia de Manabí.

\section{Salud}

La definición más importante e influyente en la actualidad con respecto a lo que por salud se entiende, sin lugar a dudas que es la de la Organización Mundial de la Salud, plasmada en el preámbulo de su Constitución "se define la salud como el estado de pleno bienestar físico, mental y social, y no solamente la ausencia de enfermedad.” (OMS 1946)

A pesar de que fue oficializada hace unos 60 años, esta definición es relevante por su institucionalidad, pues es la que sirve de base para el cumplimiento de las competencias de la OMS, que es el máximo organismo gubernamental mundialmente reconocida en materia de salud y uno de los principales actores en dicha materia. En tal sentido, la OMS viene a ser un ente internacional que da pie para que dentro de muchos Estados Nacionales se tomen medidas de políticas públicas sanitarias gubernamentales para mejorar las condiciones de vida de sus ciudadanos. De ahí la necesidad de un Estado Social que regule de manera coordinada las políticas de salud pública para evitar males mayores que los ciudadanos sin un Estado promotor de mejoras sociales sería incapaz de lograr por sí mismo.

Uno de los propósitos de la OMS es el de difundir su definición de salud para crear una delimitación homogénea de alcance global. Esta definición ha sido acogida a nivel nacional por casi todos los países, incorporándola en la legislación interna de cada uno de ellos. Esta homogeneización también se aplica con respeto al estudio de las enfermedades en vista de que las mismas trascienden las fronteras de los países, motivo por el cual se creó la Clasificación Internacional de las Enfermedades (CIE) por parte de la OMS, la cual es revisada y actualizada periódicamente.

La idea de lo que se entiende por salud depende de la interacción de múltiples factores sociales, políticos, económicos, culturales y científicos.

"La salud es una síntesis; es la síntesis de una multiplicidad de procesos, de lo que acontece con la biología del cuerpo, con el ambiente que nos rodea, con las relaciones sociales, con la política y la economía internacional" (Castro, Rincón, and Gómez 2017:147). 
Todos los procesos anteriores no están aislados ni son independientes, sino que se imbrican unos con otros, por lo que la salud depende en último término de la capacidad de controlar la interacción entre el medio físico, el espiritual, el biológico y el económico y social. Es por ello que la salud es un fenómeno social que sólo puede ser explicado teniendo en cuenta que se trata de una estructura de alto grado de complejidad como son los hechos humanos donde entra en acción una elevada cantidad de variables con fuerte interacción entre ellas.

La idea de salud responde además a una condición histórica, pues en cada momento, determinada sociedad puede tener una visión particular de la misma. De hecho, cada época corresponde a cierta estructura irrepetible de poder, técnicas, ideas, valores, costumbres, creencias y normas, de manera que la noción de lo que se entiende por salud es un producto o construcción social, así por ejemplo, la percepción del estatus y el prestigio de los médicos en las sociedades occidentales en pleno siglo $\mathrm{XX}$, considerada como hecho social inmaterial, difería mucho de los profesionales de la medicina que daban los primeros pasos hacia la consolidación de la disciplina como ciencia en la Europa de los siglos XVII y XVIII.

Cuando en el viejo continente estaban en pleno desarrollo los avances y descubrimientos del método científico, la condición de estar sano o enfermo era predominantemente atribuible a los designios divinos de corte netamente mágico-religioso. La salud y la enfermedad no se consideraban en el mayor de los casos relacionadas con algún organismo microbiológico o con factores hereditarios, psicológicos o sociales. En esos tipos de sociedades, los charlatanes, magos y brujos eran quienes socialmente tenían la función de prescribir remedios para los malestares y proporcionar la cura a los enfermos. El profesional de bata blanca, que actualmente está en los consultorios y hospitales modernos, estaba lejos de entrar en escena y ser socialmente legitimado para desempeñar el oficio de la medicina, cumpliendo con la función social de determinar quién se encuentra sano o enfermo (Cogollo-Milanes et al. 2017).

\section{Enfermería}

Florence Nightingale, nació en Florencia, Italia el 12 de mayo de 1820 y es considerada una de las pioneras en la práctica de la enfermería. Se le considera la madre de la enfermería moderna y creadora del primer modelo conceptual de enfermería. Ella definió la enfermedad como el camino que utiliza la naturaleza para desembarazarse de los efectos o condiciones que han interferido en 
la salud, y definía salud diciendo que: "es no solamente estar bien, sino ser capaz de usar bien toda la energía que poseemos" (Sarmadi 2014:32). Se rebeló contra los prejuicios de su época y contra el destino de la mujer, el de permanecer en el hogar y aplicarse solamente a tareas domésticas, eligiendo la profesión de enfermera. Su mayor éxito fue su participación en la guerra de Crimea. Un informe suyo acerca de las condiciones de vida de los soldados heridos tanto en el campo de batalla, como en los hospitales a los que eran enviados, impulsó grandes cambios en la salud pública de Europa y del mundo. Ella y sus compañeras reformaron y limpiaron el hospital, e hicieron caer la tasa de mortalidad.

La enfermería como ocupación, tiene una presencia muy antigua en el continente, al igual que en el mundo entero. En los primeros tiempos, las labores de enfermería eran ejecutadas por gente de buen corazón, sin alguna preparación; sólo en ciertos asilos se contaba con presencia de hermanas que sin ser enfermeras, al menos conocían más el oficio y demostraban constancia y disciplina. En algunos países, todavía siguen prevaleciendo estas congregaciones que se ocupan de prodigar salud a los menesterosos, ancianos o huérfanos. La enfermería surgió como profesión cuando las enfermeras graduadas de la universidad, fueron ocupando los puestos que la disminución de las congregaciones religiosas iban dejando, lo cual se veía que la tradición religiosa era muy antigua y acentuada. Tanto que hasta la actualidad, esta herencia religiosa continúa siendo significativa y decisiva en algunas instituciones que se dedican al cuidado de la salud.

La OPS en conjunto con las autoridades nacionales de salud acordaron preparar dos tipos de personal de enfermería: la enfermera graduada o titulada y la auxiliar de enfermería. Así como el decenio de 1850 - 1859, apareció la enfermera. Fue hasta los años 1900 y 1930 que la salud toma un enfoque sanitario. "En este contexto aparecen las escuelas de enfermería y después de la segunda guerra mundial se intensifica la creación de escuelas de enfermería, pero a pesar de la creación de escuelas de enfermería la pobre economía de los países impide el gran despliegue de la profesión, con un aumento de practicantes o auxiliares para atender la salud de la población” (Reyes 2015).

La Enfermería se ha incorporado a la Historia de la Salud, de forma especial desde los tratamientos de cuidados, desde el análisis de la evolución de la profesión, o desde el análisis de la relación entre la enfermería y los pacientes y la sociedad, si bien es cierto que lo ha hecho en unas fechas muy recientes. Y ello es así porque para que la investigación se pueda desarrollar no sólo debe existir, 
que entre los enfermeros y enfermeras haya existido en una mayor o menor medida un interés por la temática, sino que deben existir las condiciones de un reconocimiento de dicha investigación.

El análisis de la práctica enfermera en la Historia, por lo general, comprende cuatro etapas, que han sido delimitadas de una forma relativamente sencilla: doméstica, vocacional, técnica y profesional. Sin embargo, por mucho que alguna investigación pudiera haber en la fase vocacional, y sobre todo en la técnica, el reconocimiento tan sólo puede llegar con la etapa profesional.

\section{Indicadores de Salud}

Es ya una práctica común, al menos en las sociedades occidentales desarrolladas, evaluar la calidad de los servicios sanitarios públicos y privados mediante la medición del nivel de satisfacción de sus usuarios. El interés por conocer la opinión de los usuarios tiene, al menos, una triple justificación: primero, desde la óptica de la participación social, los ciudadanos son parte integral y central del sistema, participando activamente tanto en la evaluación como en la planificación y redefinición de la política sanitaria. Segundo, los usuarios pueden monitorizar y juzgar la calidad del servicio, aportando información a gestores públicos sobre determinados aspectos que no es posible obtener por otros medios. Y, finalmente, el objetivo de medir la satisfacción de los usuarios de los servicios, ya que un paciente satisfecho es más propenso a seguir las recomendaciones médicas y terapéuticas.

Un indicador es una construcción teórica concebida para ser aplicada a un colectivo y producir un valor numérico, por medio del cual se procura cuantificar algún concepto o noción asociada a ese colectivo. Es decir, un indicador es una operacionalización cuantitativa (números) que permite medir conceptos abstractos como calidad y eficiencia, estableciendo comparaciones en tiempo y espacio.

(Coronado et al. 2013) Se define a la eficiencia como el máximo resultado posible de productividad que puede ser alcanzado a partir de un volumen de recursos determinados, mientras que la eficacia sería la expresión general del efecto de determinada acción cuyo objetivo fuera perfeccionar la atención médica. Y la efectividad sería, a su vez, la medida de impacto que dicho procedimiento tiene sobre la salud de la población. Un indicador debe cumplir al menos con cuatro criterios básicos (Coronado et al. 2013): 
1. Validez: reflejar el aspecto de calidad para el que se creó o estableció.

2. Confiabilidad: Brindar el mismo resultado en iguales circunstancias.

3. Comprensibilidad: Fácil entendimiento del aspecto que pretende reflejar.

4. Sencillez: Facilidad para administrar, aplicar y explicar.

Otro tipo de indicadores que pueden llegar a utilizarse en la evaluación de la calidad de la atención médica, como propone la ISO 90006, son los indicadores centinela, los cuales se caracterizan por una baja probabilidad de ocurrencia y una alta probabilidad de ser producto de falla en la atención. Los indicadores de datos agregados miden el desempeño con base en eventos que ocurren con cierta frecuencia, e indican la necesidad de una revisión detallada sólo si la proporción de casos sobrepasa un límite considerado aceptable. También existen los indicadores trazadores, con una condición diagnóstica típica de una determinada especialidad médica o institución, que reflejan la globalidad de la asistencia brindada (Coronado et al. 2013).

\section{Calidad en los Servicios de Salud}

La calidad en los servicios de salud ha sido un tema ampliamente tratado en la literatura desde hace más de 40 años, su definición, interpretación y evaluación continua siendo polémica y compleja, debido a los múltiples factores involucrados en su constructo. La satisfacción de los usuarios externos, que expresa un juicio de valor individual y subjetivo, es el aspecto clave en la definición y valoración de la calidad, y es el indicador más usado para evaluar la calidad de atención en los servicios de salud y su medición se expresa en los niveles de satisfacción del usuario. Una característica fundamental de la calidad en los servicios, reconocida por (Carman 1988; Losada and Rodríguez 2007; McDougall and Levesque 1995) "que es de carácter multidimensional, aunque sin consenso respecto a las dimensiones que deben ser parte de su constructo”.

La calidad en los servicios de la salud, sea ambulatorio u hospitalario se trata, por lo tanto, "de un proceso de evaluación del desempeño que compara un resultado obtenido con criterios preestablecidos para hacer juicios de valor según se la magnitud de la diferencia" (Álvarez 2015). El concepto de calidad desde el paraje de vista técnico, es el realizado por el trabajo de la ISO, donde se mejora la definición de calidad como "grado en el que un conjunto de características inherentes cumple con los requisitos" (ISO 9000: 2005 2000); otro de los conceptos de calidad, 
“como uno de los esquemas integrales de gestión más exitosos de los últimos veinte años, evolucionando como teoría o práctica de la administración de las organizaciones, desde las primeras décadas del siglo XX" (Deming 1989).

La encuesta SERVQUAL diseñada por Parasuraman et al, por su validez y confiabilidad, es la herramienta multidimensional más aceptada y utilizada para medir la calidad de atención en empresas de servicios: validaron un nuevo constructo del SERVQUAL para hospitales. En este medio, la encuesta SERVQUAL ha sido ampliamente utilizada en diferentes servicios de salud aunque con resultados contradictorios, debido a que los estudios que aplicaron la encuesta con modificaciones en contenido y constructo, no demuestran en su metodología el proceso de validación del nuevo constructo.

Las encuestas de satisfacción de usuarios externos, requieren de una serie de propiedades psicométricas que garanticen su fiabilidad y validez, por lo tanto, es necesario contar con encuestas validadas, confiables y de fácil aplicación para medir el nivel de satisfacción e identificar las principales causas de insatisfacción de los usuarios externos en servicios de salud y que permitan implementar acciones de mejora.

Para muchos estudiosos del tema, en la última década del Siglo XX se suscitó un crecimiento vertiginoso del interés por la calidad de la atención médica en México y en muchos otros países, cuyo origen es probablemente una mayor conciencia de que, en el mundo contemporáneo, la calidad se ha convertido en un requisito indispensable de sobrevivencia económica y, para algunos afortunadamente también, de responsabilidad social y de integridad moral.

Los estudios sobre calidad de la atención que ya se venían desarrollando, mantienen continuidad y se incorporan nuevos proyectos.

\section{Modelos de Evaluación de Calidad}

Se encuentran en la literatura diversas perspectivas que caracterizan la postura de algunos autores para definir calidad del servicio, y a partir de los cuales se han diseñado diversos modelos de evaluación considerando para ello los atributos valorados en la prestación de un servicio excelente. Estos están determinados por las perspectivas desde las cuales se formulan, siendo dos las 
encontradas en la literatura, a saber: la perspectiva Expectativas-Percepciones y la perspectiva Objetiva-Subjetiva (también llamada Interno-Externo).

\section{Perspectiva Expectativas - Percepciones}

Para la definición de calidad del servicio, según la perspectiva Expectativas-Percepciones, se identifican dos posturas de los autores al respecto:

1. Uso de las expectativas y percepciones del cliente. La calidad del servicio (CS) está basada en el paradigma de la desconfirmación, como resultado de la discrepancia entre percepciones $(\mathrm{P})$ y expectativas (E) respecto al desempeño del servicio. En esta perspectiva se ubican, entre otros:

a. El modelo nórdico de calidad del servicio desarrollado por Grönroos (1984),

b. El norteamericano Service Quality (SERVQUAL) propuesto por Parasuraman y otros $(1985,1988)$,

c. El de servicios de transporte aéreo (Robledo, 2004) y el de servicios bibliotecarios LibQUAL (Cook y otros, 2001).

2. Uso de sólo las percepciones del cliente. Esta postura defiende la superioridad de la evaluación de la calidad del servicio basada sólo en las percepciones del cliente, frente a los que emplean las diferencias entre percepciones y expectativas (Cronin y Taylor, 1992; Brady y Cronin, 2001; Martínez y Martínez, 2010). Esta postura considera que incluir las expectativas en la evaluación no es necesario, debido a que ésta se fundamenta sólo en la percepción del resultado del servicio. En este sentido se han desarrollado escalas como:

a. El SERVPERF (Cronin y Taylor, 1992) y

b. El Modelo Jerárquico Multidimensional (Brady y Cronin, 2001).

\section{Perspectiva Objetiva - Subjetiva}

Respecto a la perspectiva Objetiva-Subjetiva (Interna-Externa) para la definición de la calidad del servicio, se identifican dos puntos de vista.

1. La externa, asociada a la percepción y/o expectativas del cliente, también catalogada como calidad subjetiva; y 
2. El punto de vista interno cuya evaluación se realiza considerando al prestador del servicio, el cual usa indicadores internos u objetivos (Ruiz, 2001; Duque, 2005), como por ejemplo el modelo de evaluación de la calidad del transporte público terrestre UNE-EN 13816 (Ministerio de Fomento de España, 2006; Ministerio de Transporte y Telecomunicaciones de Chile, 2006).

Se describen a continuación algunos de los modelos de mayor referencia en la literatura científica, considerando los cuestionamientos publicados a los mismos, sus aplicaciones, o por sus contribuciones (Parasuraman, Zeithaml, and Berry 1988), el Modelo Service Performance (SERVPERF) de (Cronin and Taylor 1992), el Modelo Jerárquico Multidimensional de Brady y Cronin en 2001, el modelo para servicios bibliotecarios LibQUAL propuesto por (Cook et al. 2001), el E-S-QUAL sobre calidad del servicio entregado por sitios Web de Parasuraman, Zeithaml y Malhotra en el 2005 y, finalmente un modelo de Evaluación unidimensional de la calidad del servicio propuesto por Martínez y Martínez del 2010.

Para evaluar la calidad en los servicios de salud, se tiene como referencia obligatoria la propuesta del médico (Donabedian 1966) establece las dimensiones de estructura, proceso y resultado, además de sus respectivos indicadores para evaluarla. Esta relación entre proceso y resultado, y la sistematización de criterios, genera reflexiones importantes sobre la responsabilidad en la calidad de este tipo de servicio. En este modelo, la estructura describe las características físicas, de organización y otros rasgos del sistema asistencial y de su entorno; el proceso es lo que se hace para tratar a los pacientes, finalmente el resultado es lo que se consigue, normalmente traducido en una mejora en la salud, aunque también en la actitud, los conocimientos y en la conducta adoptada para una salud futura.

"El significado de la calidad y de los procedimientos empleados para evaluarla, dependería de la política que se adoptara con respecto a la legitimidad de la medicina" (Donabedian 1984). Y la calidad puede medirse en dos dimensiones interrelacionadas e interdependientes: la técnica y la interpersonal. La atención técnica hace insistencia a la atención de la ciencia y la tecnología médica de manera que rinda un enorme beneficio para la salud, sin aumentar con ello sus riesgos, en cambio la atención interpersonal mira el grado de apego a valores, normas, expectativas y aspiraciones de los pacientes. 
"La inhabilidad de los pacientes para juzgarla calidad técnica de los servicios de salud hace que su evaluación se fundamente en diversos aspectos de la relación interpersonal, el desempeño funcional del proveedor y los factores ambientales que rodean la prestación del servicio" (Losada and Rodríguez 2007).

"El modelo SERVQUAL es uno de los instrumentos más utilizados en el sector servicios para evaluar el nivel de calidad percibida por los clientes" (Parasuraman, Zeithaml, and Berry 1988). Es uno de los instrumentos más citados en la literatura sobre calidad y el que mayor atención ha recibido por parte de autores de muy distintas disciplinas. Su estructura de 44 ítems, divididos en dos escalas de 22 ítems cada una, evalúa, por un lado, las expectativas sobre el servicio ideal y, por otro, las percepciones de los clientes del servicio que han recibido.

El modelo SERVQUAL "se basa en la idea de que la calidad vista por el cliente es el resultado de la diferencia entre expectativas y percepciones, el denominado paradigma de la desconfirmación, que hasta la fecha es la teoría con mayor solidez" (Oliver 1993). Sin embargo, pese al gran interés que ha despertado este instrumento, no está exento de ciertos problemas metodológicos. Por ejemplo, no se ha podido corroborar la estructura de cinco factores sugerida por sus autores; "la escala de expectativas no ha demostrado suficiente validez predictiva, la versión ponderada no ha supuesto ventaja metodológica alguna y se ha demostrado que la escala de percepciones parece reunir mayor validez discriminante y mayor capacidad predictiva que la puntuación SERVQUAL" (Cronin and Taylor 1992), calculada a partir de la diferencia entre expectativas y percepciones.

"En el sector sanitario SERVQUAL ha despertado también muchas expectativas, ya que ha sido considerado como una alternativa que reúne ventajas teóricas y prácticas frente a las habituales encuestas de posthospitalización" (Saleh and Ryan 1991). Sin embargo, también en su aplicación al medio sanitario se han resaltado los mismos inconvenientes.

Los autores (Parasuraman, Zeithaml, and Berry 1988) sugirieron diferentes alternativas a su cuestionario SERVQUAL con la intención de superar las principales críticas. En esta línea, "rediseñaron su cuestionario y sugirieron una forma alternativa de respuesta combinando expectativas y percepciones en una misma escala" (Mira et al. 1998). 
El modelo SERQUAL Asemeja las cinco dimensiones relativas a los criterios de evaluación que utilizan los clientes para valorar la calidad en un servicio.

1. Fiabilidad: Habilidad para realizar el servicio de modo cuidadoso y fiable.

2. Capacidad de Respuesta: Disposición y voluntad para ayudar a los usuarios y proporcionar un servicio rápido.

3. Seguridad: Conocimientos y atención mostrados por los empleados y sus habilidades para concitar credibilidad y confianza.

4. Empatía: Atención personalizada que dispensa la organización a sus clientes.

5. Elementos Tangibles: Apariencia de las instalaciones físicas, equipos, personal y materiales de comunicación.

Estas cinco dimensiones de la calidad de servicio son evaluadas mediante el cuestionario SERVQUAL.

\section{El cuestionario SERVQUAL}

El cuestionario SERVQUAL consta de tres secciones:

En la primera se interroga al cliente sobre las expectativas que tiene acerca de lo que un servicio determinado debe ser. Esto se hace mediante 22 declaraciones en las que el usuario debe situar, en una escala de 1 a 7 , el grado de expectativa para cada una de dichas declaraciones.

En la segunda, se recoge la percepción del cliente respecto al servicio que presta la empresa. Es decir, hasta qué punto considera que la empresa posee las características descritas en cada declaración.

Finalmente, otra sección, situada entre las dos anteriores, cuantifica la evaluación de los clientes respecto a la importancia relativa de los cinco criterios, lo que permitirá ponderar las puntuaciones obtenidas.

Las 22 declaraciones que hacen referencia a las cinco dimensiones de evaluación de la calidad citadas, son agrupadas de la siguiente manera:

- $\quad$ Elementos tangibles: Ítems de 1 al 4.

- $\quad$ Fiabilidad: Ítems del 5 al 9. 
- $\quad$ Capacidad de respuesta: Ítems del 10 al 13.

- $\quad$ Seguridad: Ítems del 14 al 17.

- $\quad$ Empatía: Ítems del 18 al 22.

De esta manera, el modelo SERVQAL de Calidad de Servicio permite disponer de puntuaciones sobre percepción y expectativas respecto a cada característica del servicio evaluada. La diferencia entre percepción y expectativas indicará los déficits de calidad cuando la puntuación de expectativas supere a la de percepción.

El modelo SERVQUAL de Calidad de Servicio muestra la línea a seguir para mejorar la calidad de un servicio y que, fundamentalmente, consiste en reducir determinadas discrepancias. En primer lugar, se asume la existencia de cinco deficiencias en el servicio. Cada una asociada a un tipo de discrepancia.

Se denomina deficiencia 5 a la percibida por los clientes en la calidad de los servicios. En otras palabras, esta deficiencia representa la discrepancia existente, desde el punto de vista del cliente, entre el servicio esperado y el servicio recibido. Se trata de la deficiencia fundamental ya que define la calidad del servicio.

En el Modelo SERVQUAL de Calidad de Servicio, las otras cuatro deficiencias se refieren al ámbito interno de la organización y serán las responsables de la aparición de la deficiencia 5:

Deficiencia 1: Discrepancia entre las expectativas de los clientes y las percepciones de los directivos. Si los directivos de la organización no comprenden las necesidades y expectativas de los clientes, no conocen lo que éstos valoran de un servicio, difícilmente podrán impulsar y desarrollar acciones que incidan eficazmente en la satisfacción de necesidades y expectativas. Más bien al contrario, con una idea equivocada los directivos iniciarán actuaciones poco eficaces que pueden provocar una reducción de la calidad misma.

Deficiencia 2: Discrepancia entre las percepciones de los directivos y las especificaciones de las especificaciones y normas de calidad. Aunque los directivos comprendan las necesidades y expectativas de los clientes de la organización, su satisfacción no está asegurada. Otro factor que debe estar presente, para alcanzar una alta calidad, es la traducción de esas expectativas, conocidas y comprendidas, a especificaciones o normas de calidad del servicio.

\section{0}

Pol. Con. (Edición núm. 33) Vol. 4, No 5, mayo 2019, pp. 204-247, ISSN: 2550 - 682X 
Deficiencia 3: Discrepancia entre las especificaciones de la calidad del servicio y la prestación del servicio. No basta con conocer las expectativas y establecer especificaciones y estándares. La calidad del servicio no será posible si las normas y procedimientos no se cumplen. Este incumplimiento puede ser debido a diversas causas, como empleados no capacitados adecuadamente, falta de recursos o procesos internos mal diseñados.

Deficiencia 4: Discrepancia entre la prestación del servicio y la comunicación externa. Para el modelo SERVQUAL de calidad de servicio, uno de los factores clave en la formación de expectativas sobre el servicio, por parte del cliente, es la comunicación externa de la organización proveedora. Las promesas que ésta hace y la publicidad que realiza afectarán a las expectativas. De este modo, si no van acompañadas de una calidad en la prestación consistente con los mensajes, puede surgir una discrepancia expectativa - percepción. Esta discrepancia puede reducirse mediante la coordinación de las características de la prestación con la comunicación externa que la organización hace.

El Modelo SERVQUAL de Calidad de Servicio no representa únicamente una metodología de evaluación de la calidad de servicio. Constituye en sí un completo enfoque para la mejora de la calidad de servicio.

\section{EI Modelo SERVQHOS}

\section{Instrumento}

Con el propósito de medir la calidad en los servicios hospitalarios, se ha adaptado la versión de Servqual (Parasuraman, Zeithaml, \& Berry 1988), que considera mayor aporte de información para la gestión de mejoramiento de la calidad. En esta reformulación se determinó la validez, consistencia interna, aditividad y los elementos de cada escala con el fin de construir un instrumento que tuviese un mejor comportamiento metodológico. Este instrumento adaptado se denominó SERVQHOS, y fue diseñado por los españoles (Mira Solves et al. 1997), el cual considera un nuevo formato incorporando los elementos sugeridos por (Parasuraman, Zeithaml, and Berry 1988). El instrumento es validado, lo que permitió comprobar su consistencia, validez de construcción, capacidad discriminante y su capacidad predictiva respecto a una medida global de satisfacción del paciente, la herramienta reúne las suficientes condiciones psicométricas para ser utilizada como medida de la capacidad percibida por el paciente en el medio hospitalario. 
Conserva el sentido del cuestionario original a partir de la teoría de las discrepancias entre expectativas y percepciones y es utilizado frecuentemente para realizar intervención y mejoras en el proceso de asistencia sanitaria. No pretende invertir el orden en las prioridades asistenciales, pero sí buscan ayudar a resolver el difícil reto de saber responder a esas necesidades de salud, siendo capaces de satisfacer las expectativas de los pacientes, el cuestionario SERVQHOS está diseñado a partir de la teoría de la discrepancia entre expectativas y percepciones y se utiliza para determinar la calidad percibida en la atención hospitalaria. Está organizado en cuatro partes:

- $\quad$ Primero se describe los objetivos y la importancia de la información solicitada al usuario. Establece aspectos éticos como el permiso y el consentimiento para su aplicación; determina que la información será manejada de forma anónima y confidencial.

- Segundo, consta de 19 ítems que se evalúan mediante una escala de Likert de cinco puntos, en la cual indaga al usuario por aspectos tangibles e intangibles de la calidad de la atención y los criterios basados en las expectativas y percepciones para la evaluación, que van de uno a cinco, siendo 1 "mucho peor de lo que esperaba" y 5 "mucho mejor de lo que esperaba".

- Tercero, es una encuesta adicional que indaga sobre la satisfacción global del usuario y aspectos relacionados con la misma.

- Cuarto, donde se indaga por los datos sociodemográficos de los usuarios que diligencian el cuestionario, como sexo, edad, estado civil, nivel de estudios y ocupación. Existe un espacio en blanco adicional en el cual el usuario puede escribir las sugerencias que tenga para el mejoramiento de la calidad de la atención hospitalaria.

\section{EI Modelo SERVQHOS-E}

El modelo SERVQHOS es útil para analizar la calidad de la atención hospitalaria, sin embargo, en el campo laboral de enfermería a nivel profesional y técnico constituye, en gran proporción, los servicios de salud prestados en este tipo de instituciones, fundaciones; los profesionales de enfermería, así como las auxiliares constituyen un gran porcentaje del recurso humano disponible para brindar los servicios de salud, desde el ingreso del usuario hasta su egreso, razón por la cual 
es el contacto permanente y progresivo, punto importante y crítico de la evaluación asistencial, lo cual se traduce en la calidad de la atención del servicio que se brinda.

El instrumento SERVQHOS-E está basado en la teoría de la discrepancia entre expectativas y percepciones, mencionado anteriormente y es utilizado para evaluar la calidad percibida de la atención de enfermería por parte del usuario. El cuestionario SERVQHOS-E cuenta con un consentimiento informado y sigue las normas éticas de investigación y lo dispuesto en las normas científicas, técnicas y administrativas para la investigación en salud con seres humanos, que implicó un bajo riesgo para los participantes. El instrumento consta de 16 ítems, de los cuales 12 corresponden a calidad subjetiva y permiten analizar la percepción que tiene el usuario respecto a la atención; también, contempla 4 ítems de calidad objetiva aspectos tangibles durante la estancia hospitalaria. Los ítems de calidad subjetiva y objetiva son calificados a través de una escala tipo Likert de 1 a 5; es decir, que califica de 1 "mucho peor de lo que esperaba" a 5 "mucho mejor de lo que esperaba" (Barragán and Manrique-Abril 2010).

La segunda parte del instrumento contiene una pregunta que indica el nivel de satisfacción global, luego aborda 5 preguntas las variables sociodemográficas.

Elementos que conforman la Calidad de Atención en Enfermería

En enfermería específicamente, la prestación de servicios hace parte de la atención hospitalaria y es un eje fundamental en la actividad profesional; constituye un proceso basado en una relación enfermera-usuario, el cual es permanente, dinámico y directo. En este proceso, intervienen conocimientos, recursos, infraestructura y procedimientos como parte de la atención general, también de aspectos personales subjetivos como la cordialidad, la empatía y la credibilidad (Swan, Carroll, and Bloomington 1980).

\section{Calidad Objetiva}

Conformado por los ítems que son visibles y palpables por el usuario en la prestación de un servicio: como la tecnología de los equipos, la apariencia del personal de enfermería, señalizaciones para orientarse y el estado de las habitaciones. En el estudio realizado por (Barragán, Pedraza, and Rincón 2013:192) se encontró que "La apariencia del personal de enfermería” recibió la calificación más alta. Resultados similares se encontraron en investigaciones realizadas en la 
región en hospitales públicos de Boyacá, lo cual significa que los usuarios perciben la calidad cercana a un resultado de "mejor de lo que esperaba". A nivel internacional, encontraron medias por encima de 4 en uno de los hospitales, lo cual indica que la apariencia del personal de enfermería, es importante al momento de emitir un juicio sobre la calidad objetiva de la atención de dicho personal. Para los usuarios es importante la presentación del personal en el momento que realiza una actividad o procedimiento y que es necesario mejorar el aspecto físico de las habitaciones; sin embargo, se observa que en el transcurso del tiempo y los resultados del estudio realizado en el mismo hospital, la percepción de éstas variables ha mejorado.

Los resultados de la calidad objetiva y subjetiva según estudio realizado por Barragan y Moreno (2012), evidencian una puntuación media de 3.61 para todos los ítems, la cual es estadísticamente significativa $(\mathrm{p}=0.000)$ dadas las diferencias de puntuación entre la primera y segunda medición, que fueron mayores en la primera para los hospitales A y C y menores en el hospital B. De igual forma, se encontraron diferencias significativas de puntuación, entre la primera y segunda medición por hospital con tendencia al aumento en la segunda, $(\mathrm{p}=0.000)$ especialmente en los hospitales A y B, el hospital C mostró una tendencia a mantener la puntuación.

En relación a los ítems que evalúan la calidad objetiva, se encuentra que la tecnología y equipos con una media de 3.54, así como el estado de las habitaciones con media de 3.63, son los ítems que menor puntuación recibieron, a diferencia de la presentación del personal e indicaciones para orientarse con una media de 3.79; los usuarios percibieron mejor este tipo de calidad en los hospitales A y C.

\section{Calidad Subjetiva}

Integrado por los ítems: el interés de las enfermeras, la información que proporcionan, el tiempo de espera para ser atendido, el interés por solucionar los problemas, la puntualidad, la rapidez con que consigue lo que necesita, la disposición para ayudar, la confianza (seguridad) que trasmiten, la amabilidad (cortesía), la preparación para hacer bien el trabajo (capacitación), el trato personalizado y la capacidad para comprender las necesidades de los pacientes el interés de las enfermeras, la información que proporcionan, el tiempo de espera para ser atendido, el interés por solucionar los problemas, la puntualidad, la rapidez con que consigue lo que necesita, la disposición 
para ayudar, la confianza (seguridad) que trasmiten, la amabilidad (cortesía), la preparación para hacer bien el trabajo (capacitación), el trato personalizado y la capacidad para comprender las necesidades de los pacientes.

\section{La Institución y el Problema}

La fundación Cottolengo se encuentra ubicada en el barrio los Geranios, calle 295 entre avenidas 235 y 236, del cantón Manta, provincia de Manabí; fue aprobada por el Ministerio de Inclusión, Económico y Social (MIES) el 23 de abril del 2012 con acuerdo ministerial \#0087 y comienza su personería jurídica. Es una fundación tripartita compuesta por una parte por la arquidiócesis de Portoviejo que representa los bienes muebles e inmuebles, por otra parte por las Hermanas de San José Benito Cottolengo de Torino - Italia, que representa a la asistencia y está a cargo del personal y por ultimo un directorio que a su vez es voluntario, se escoge y elige democráticamente cada 2 años con un máximo de reelección de un periodo, que es el representante legal/patronal.

La fundación no tiene fines de lucro y acoge a pacientes en etapa terminal de su enfermedad para sus cuidados paliativos oncológicos y no oncológicos, a pacientes post quirúrgicos para rehabilitación física y a pacientes adultos mayores con algún tipo de demencia para residencia sanitaria asistencial (asilo).

Las enfermeras auxiliares realizan turnos rotativos de la siguiente manera:

- En la mañana, desde las $07 \mathrm{~h} 00$ hasta las $13 \mathrm{~h} 00$, con la sigla M

- En la tarde, desde las $13 \mathrm{~h} 00$ hasta las $19 \mathrm{~h} 00$, con la sigla $\mathrm{T}$

- En la noche, desde las $19 \mathrm{~h} 00$ hasta la $07 \mathrm{~h} 00$ del siguiente día, con la sigla $\mathrm{N}$.

- Después de un turno de noche tienen libre las siguientes 24 horas, con la sigla L; cabe recalcar que y se reintegran con el turno de "tarde", el siguiente día "mañana", y "noche" el siguiente día y así sucesivamente se cumplen los turnos rotativos de todo el personal de enfermería.

La licenciada jefa tiene turno desde las $07 \mathrm{~h} 30$ hasta las $16 \mathrm{~h} 30$, un equipo de rehabilitación conformado por tres terapistas ocupacionales que realizan una jornada de trabajo desde las $08 \mathrm{~h} 00$ hasta las $16 \mathrm{~h} 30$ de lunes a sábado, donde se práctica la estimulación cognitiva, motricidad fina, motricidad gruesa, terapia física, electroestimulación y ultrasonido; y el equipo médico se 
encuentra solo en horas diurnas, un turno desde las $08 \mathrm{~h} 00$ hasta las $16 \mathrm{~h} 00 \mathrm{y}$ otro turno desde las 14h30 hasta las 18h30. Durante el horario diurno se mantiene cubierto con la jefa de enfermera, las auxiliares de enfermería, terapistas ocupacionales, médicos del turno diurno y una parte del voluntariado (directiva) que ayuda con algunas de las actividades de la vida diaria de nuestros pacientes (alimentación, aseo personal y traslado), se da información a todos los familiares de la evolución de sus pacientes, se explica tratamientos y se entrega recetas.

Se pasa la visita médica todos los días de lunes a sábado en el área de cuidados paliativos, se realiza curaciones, se prescribe y se baja nueva medicación, se indican tratamientos, dietas y terapias de rehabilitación física u ocupacional; se pasa médica los demás pabellones trisemanalmente dependiendo de la distribución de pacientes ya que se los va clasificando de acuerdo a su historial clínico en grave o no grave. Durante el horario nocturno solo queda cubierto con el personal de enfermería (auxiliares) una por cada pabellón, un camillero para ayudar a realizar los traslados de los pacientes y el respectivo cambio de posición, un guardia que vigilará y rondará por todos los predios de la fundación.

Durante el turno diurno se encuentra cubierto con más personal (médicos, terapistas, voluntarios y enfermera jefa) para realizar otras funciones (terapia física, alimentación, traslado, administración de medicación, etc.) que no se realizan en el turno nocturno, por eso se les indicará funciones específicas a realizar (administración de medicación, cambios posturales y baño del paciente), es aquí donde el personal médico hace un llamado de atención al personal de enfermería del turno nocturno, porque muchas veces el paciente no se encuentra debidamente bañado, hay que recalcar que los pacientes graves se les realiza baño en cama y a los pacientes no graves baño normal. O se los encuentra con úlceras de presión por no haber realizado un cambio postural adecuado y hasta pacientes que llegan a fallecer durante la noche, pacientes graves que están aparentemente estables durante el día por su producción de estos desechos tipo infecciosos.

\section{Metodología}

Para el presente trabajo de investigación se desarrolló en un estudio de tipo descriptivo de corte transversal con abordaje cuantitativo y cualitativo, para determinar la calidad de la atención de 
Enfermería desde la percepción de los pacientes hospitalizados en la Fundación Cottolengo de la Ciudad de Manta.

Los estudios descriptivos miden, de manera más bien independiente, los conceptos o variables a los que se refieren. Aunque, pueden integrar las mediciones de cada una de dichas variables para decir cómo es y cómo se manifiesta el fenómeno de interés. Su objetivo no es indicar cómo se relacionan las variables medidas, se centran en medir con la mayor precisión posible. En esta clase de estudio el investigador debe ser capaz de definir qué se va a medir y cómo lograr precisión en esa medición.

Se trata de un estudio transversal puesto que los diseños de investigación transeccional o transversal recolectan datos en un solo momento, en un tiempo único. Su propósito es describir variables y analizar su incidencia e interrelación en un momento dado. Es como tomar una fotografía de algo que sucede (Hernández, Fernández-Collado, and Baptista 1998). En este estudio se pretende determinar la calidad de la atención de Enfermería en un momento dado del tiempo, es decir, en un tiempo determinado.

Cualitativo, la investigación cualitativa es el término que se utiliza para referirse a la investigación que depende de datos recopilados en situaciones no controladas. Por lo tanto, la investigación cualitativa muestra más interés por la complejidad y los efectos de las variables que por el control de ellas, el cual es uno de los propósitos más importantes de la investigación cuantitativa. Tomando en cuenta el carácter espontáneo de muchos datos cualitativos, un amplio abanico de herramientas y técnicas han sido desarrolladas para brindar asistencia a los investigadores en su búsqueda del modo más idóneo de abordar su pregunta de investigación (Lankshear and Knobel 2000).

Una investigación cuantitativa parte de un paradigma positivista, cuyo objetivo es explicar y describir casualmente, así como generalizar, extrapolar y universalizar, siendo el objeto de esta investigación hechos objetivos existentes y sometidos a leyes y patrones generales. El fenómeno que estudia este tipo de investigación es siempre observable, medible y replicable, haciéndolo en un contexto controlado donde se utiliza un lenguaje con precisión matemática y con modelos estadísticos de la codificación numérica. En cuanto al investigador que se encarga de este tipo de estudio, debe ser un observador externo, que utiliza un método deductivo para realizar un análisis 
estadístico, dependiendo las técnicas utilizadas para el estudio del método seleccionado, pero siempre han de estar prefijadas (ACONA 2015).

\section{Universo}

El Universo está conformado por los pacientes que están ingresados en la fundación Cottolengo, de todas sus áreas, (Recuperación, Paliativa y de Asilo) que recibieron atención por el personal de Enfermería durante su estancia hospitalaria como parte de sus cuidados.

\section{Criterios de Inclusión}

- Pacientes mayores de 35 años

- Pacientes con secuelas de enfermedades cerebrovasculares

- Pacientes que tienen más de 5 días de ingreso hospitalario

\section{Fuente de información}

La fuente de información fue primaria, es decir, es suministrada por los familiares de los pacientes que se encuentran ingresados en la fundación; por presentar alguna secuela cognitiva, que no los deja tomar decisiones propias, que cumplen con los criterios de inclusión, y decidieron participar de forma voluntaria en la investigación firmando el consentimiento informado.

\section{Instrumentos de Investigación.}

Se utilizará el Instrumento SERVQHOS-E (versión para Enfermería del SERVQHOS original) como encuesta.

\section{Análisis de Resultados}

A continuación, se presentan los principales resultados obtenidos de la investigación

\section{Análisis Estadístico}

La tabla 1 presenta los resultados del sexo de los encuestados. El 55.4\% de los encuestados son mujeres mientras que el $44.6 \%$ son hombres. 
Tabla 1 Sexo de los encuestados

\begin{tabular}{llc|c|c|}
\hline & & Frecuencia & Porcentaje & Porcentaje válido \\
\hline Válido & Masculino & 29 & 44,6 & 44,6 \\
\cline { 2 - 5 } & Femenino & 36 & 55,4 & 55,4 \\
\cline { 2 - 5 } & Total & 65 & 100,0 & 100,0 \\
\hline
\end{tabular}

Fuente:

La tabla 2 presenta los resultados de la edad de los encuestados. El 55.4\% tienen más de 81 años y el $4.6 \%$ entre $71-75$ años

Tabla 2 Edad de los Encuestados

\begin{tabular}{lcc|c|c|}
\hline & & Frecuencia & Porcentaje & Porcentaje válido \\
\hline Válido & $<=65$ & 6 & 9,2 & 9,2 \\
\cline { 2 - 5 } & $66-70$ & 5 & 7,7 & 7,7 \\
\hline $71-75$ & 3 & 4,6 & 4,6 \\
\hline $76-80$ & 15 & 23,1 & 23,1 \\
\hline $81+$ & 36 & 55,4 & 55,4 \\
\hline Total & 65 & 100,0 & 100,0 \\
\hline
\end{tabular}

\section{Fuente:}

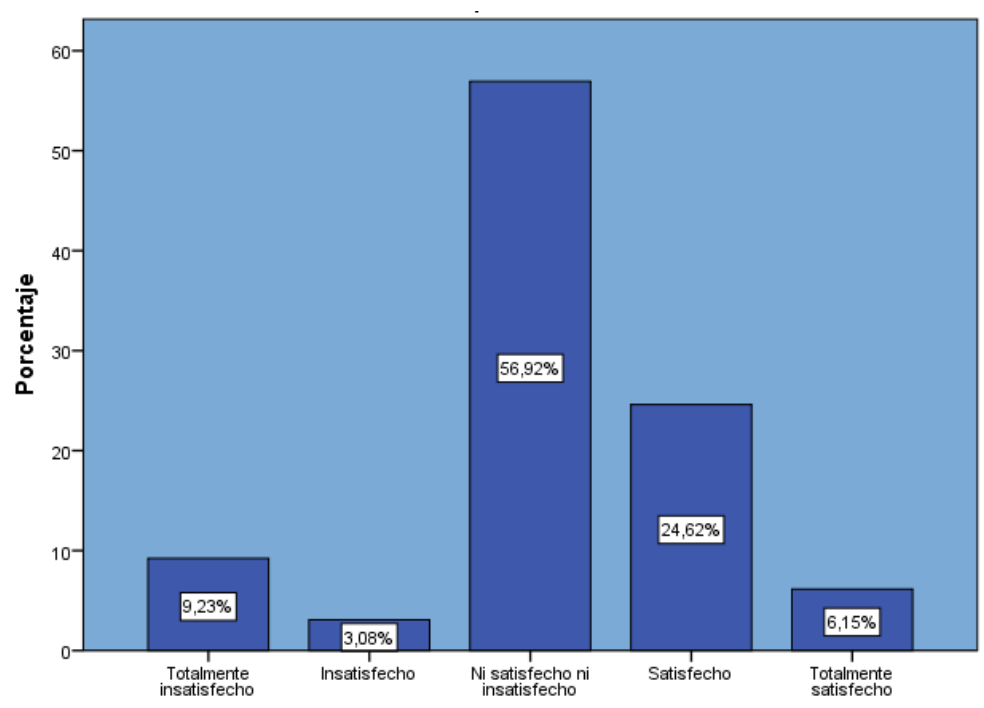

Figura 1 Transmitió confianza el personal de auxiliar de enfermera mientras lo atendía cuando estuvo ingresado en el área de hospitalización de la Fundación Cottolengo 
El $56.92 \%$ de los usuarios encuestados que estuvieron ingresados en el área de hospitalización de la Fundación Cottolengo estuvo ni satisfecho ni insatisfecho con la conducta de la enfermera que le inspira confianza, el $24.62 \%$ estuvo satisfecho, el $9.23 \%$ totalmente insatisfecho, el $6.15 \%$ totalmente satisfecho y un $3.08 \%$ insatisfecho.

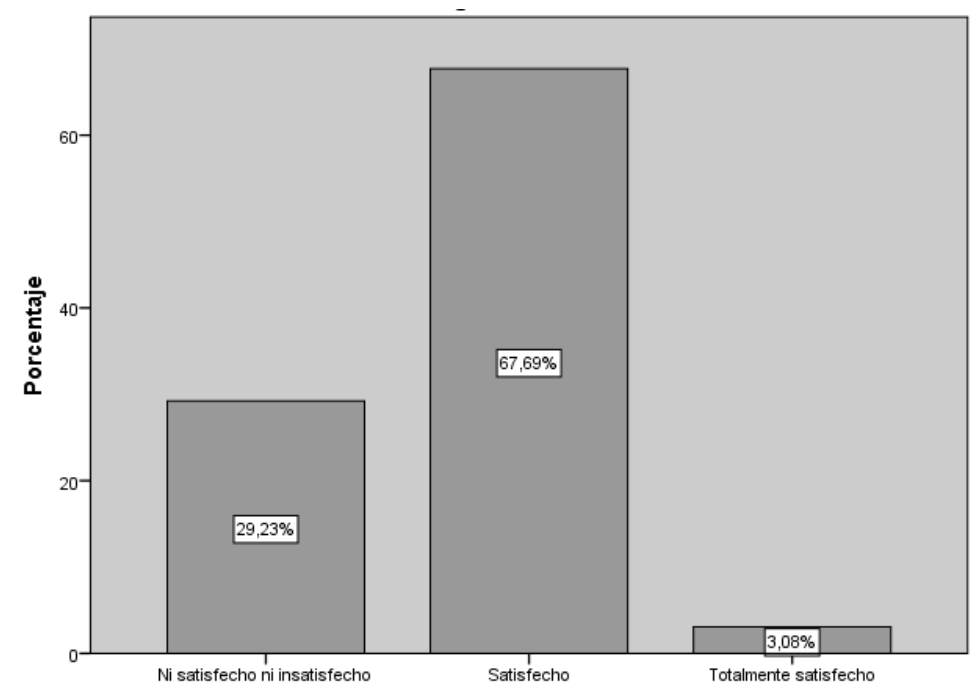

Figura 2 Residencia de los pacientes encuestados que estuvieron ingresados en el área de hospitalización de la Fundación Cottolengo

El 67.69\% de los usuarios encuestados que estuvieron ingresados en el área de hospitalización de la Fundación Cottolengo fue satisfecho con los equipos biomédicos que presentaba, el 29.23\% estuvo ni satisfecho ni insatisfecho y un 3.08\% totalmente satisfecho.

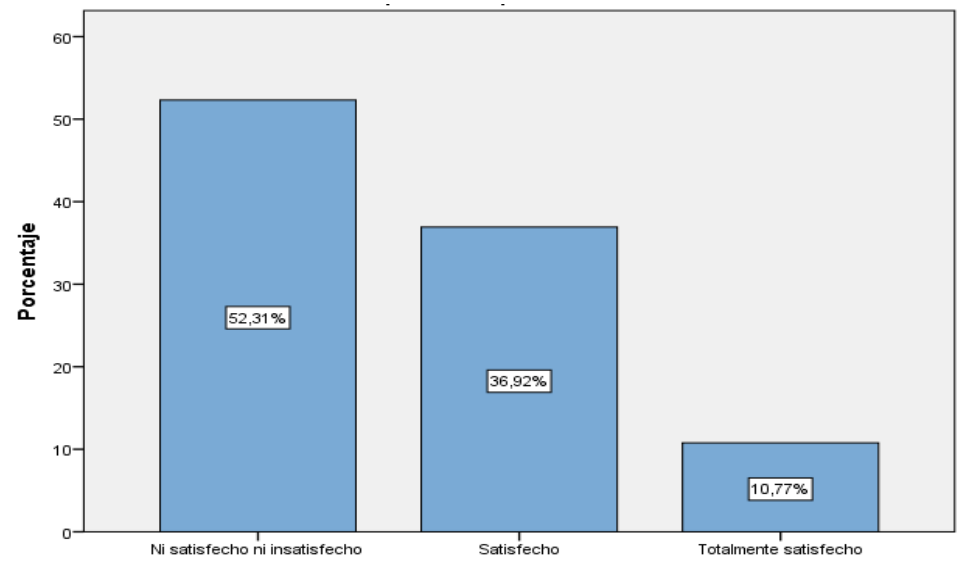

Figura 3 Como es el aspecto del personal de auxiliares de enfermería del área de hospitalización de la Fundación Cottolengo 
El 52.31\% de los usuarios encuestados que estuvo ingresado en el área de hospitalización de la Fundación Cottolengo fue ni satisfecho ni insatisfecho con el aspecto del personal de auxiliares de enfermería, el $36.92 \%$ estuvo satisfecho y un $10.77 \%$ estuvo totalmente satisfecho.

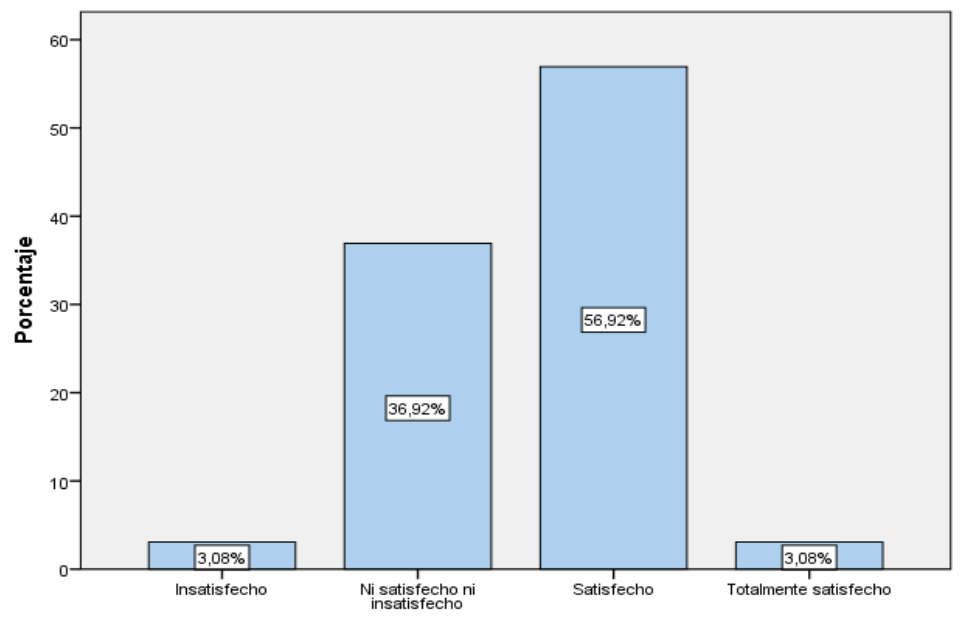

Figura 4 Qué tal le parece la señalización intrahospitalaria de la Fundación Cottolengo

El 56.92\% de los usuarios encuestados que estuvieron ingresados en el área de hospitalización de la Fundación Cottolengo estuvo satisfecho con la señalización intrahospitalaria, el 36.92\% estuvo ni satisfecho ni insatisfecho, un 3.08\% estuvo insatisfecho y en un mismo porcentaje totalmente satisfecho.

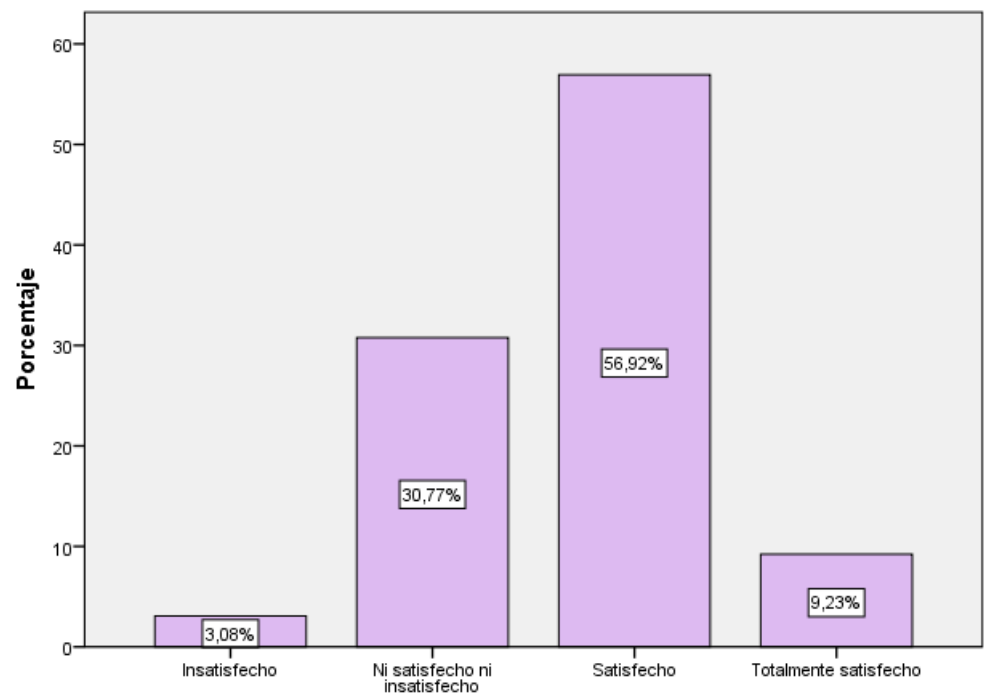

Figura 5 Qué piensa de la comodidad de las habitaciones del área de hospitalización de la Fundación Cottolengo 
El 56.92\% de los usuarios encuestados que estuvieron en el área de hospitalización de la Fundación Cottolengo fue satisfecho con la comodidad de las habitaciones, el $30.77 \%$ estuvo ni satisfecho ni insatisfecho, el $9.23 \%$ totalmente satisfecho y un 3.08\% insatisfecho.

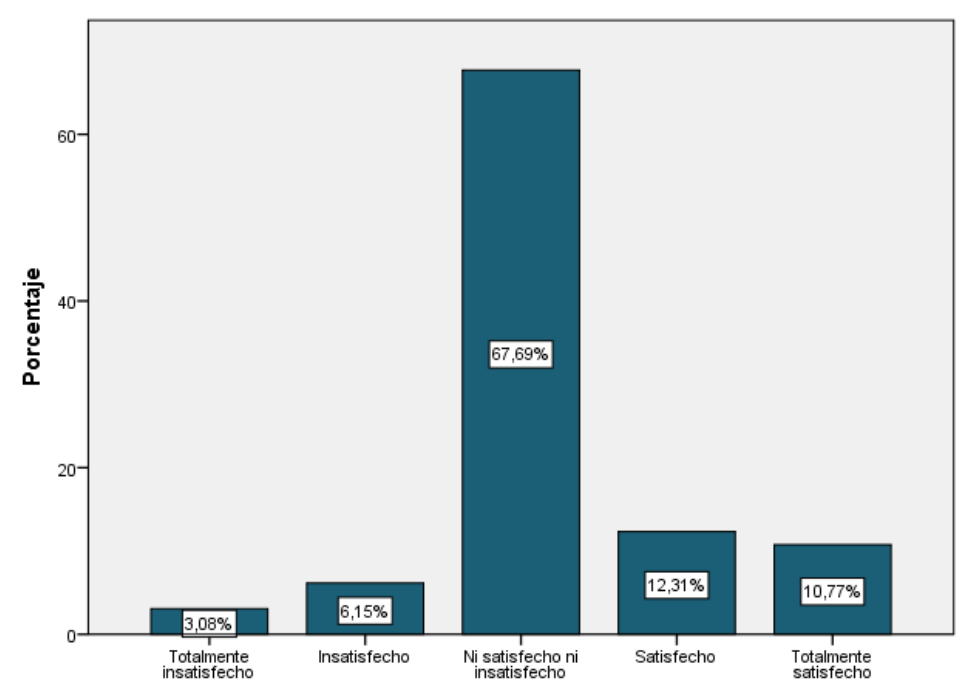

Figura 6 La información que le proporcionó el personal de auxiliar de enfermería del área de hospitalización de la Fundación Cottolengo fue la necesaria

El 67.69\% de los usuarios encuestados que estuvieron ingresados en el área de hospitalización de la Fundación Cottolengo con la información que le proporcionó el personal de auxiliar de enfermería fue ni satisfecho ni insatisfecho, el $12.31 \%$ estuvo satisfecho, el $10.77 \%$ totalmente satisfecho, el $6.15 \%$ insatisfecho y un 3.08\% totalmente insatisfecho.

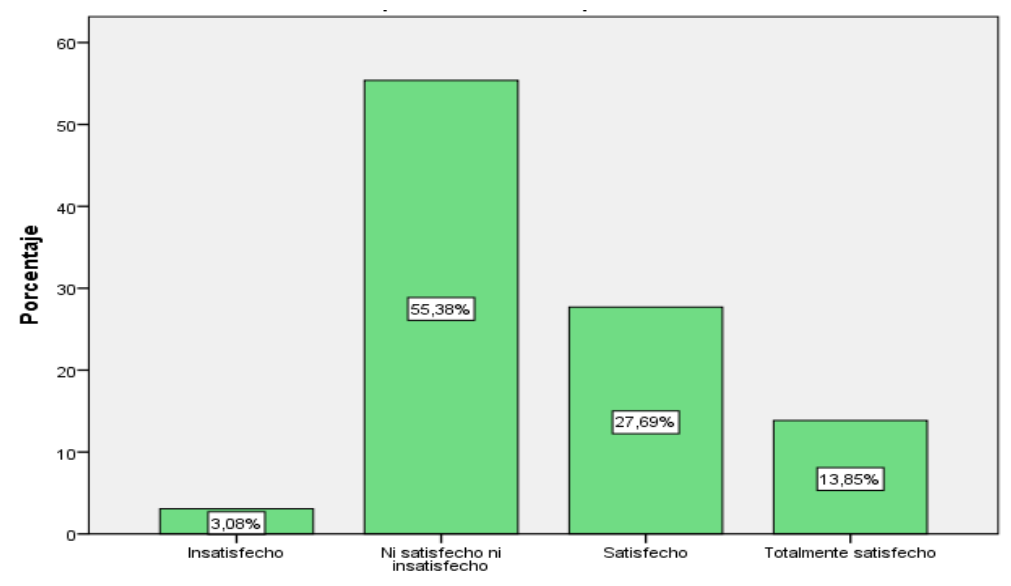

Figura 7 Presentó interés la auxiliar de enfermería en solucionar sus problemas mientras estuvo hospitalizado en la Fundación Cottolengo 
El 55.38\% de los usuarios encuestados que estuvieron ingresados en el área de hospitalización de la Fundación Cottolengo con el interés del personal de enfermería en solucionar sus problemas fue ni satisfecho ni insatisfecho, el $27.69 \%$ refiere que estaba satisfecho, el $13.85 \%$ totalmente satisfecho y un $3.08 \%$ insatisfecho.

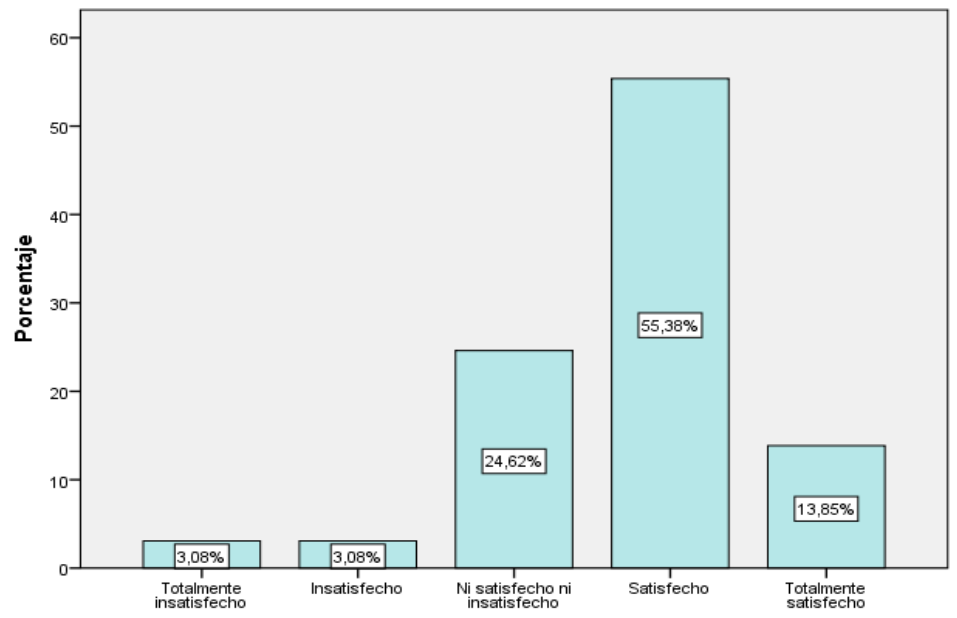

Figura 8 El auxiliar de enfermería fue puntual al momento de prestar su servicio en el área de hospitalización de la Fundación Cottolengo

El 55.38\% de los usuarios encuestados que estuvo ingresado en el área de hospitalización de la Fundación Cottolengo estuvo satisfecho con la puntualidad del personal de auxiliares de enfermería al momento de prestar su servicio, el $24.62 \%$ estuvo ni satisfecho ni insatisfecho, el $13.85 \%$ totalmente satisfecho, el 3.08\% insatisfecho y un 3.08\% totalmente insatisfecho.

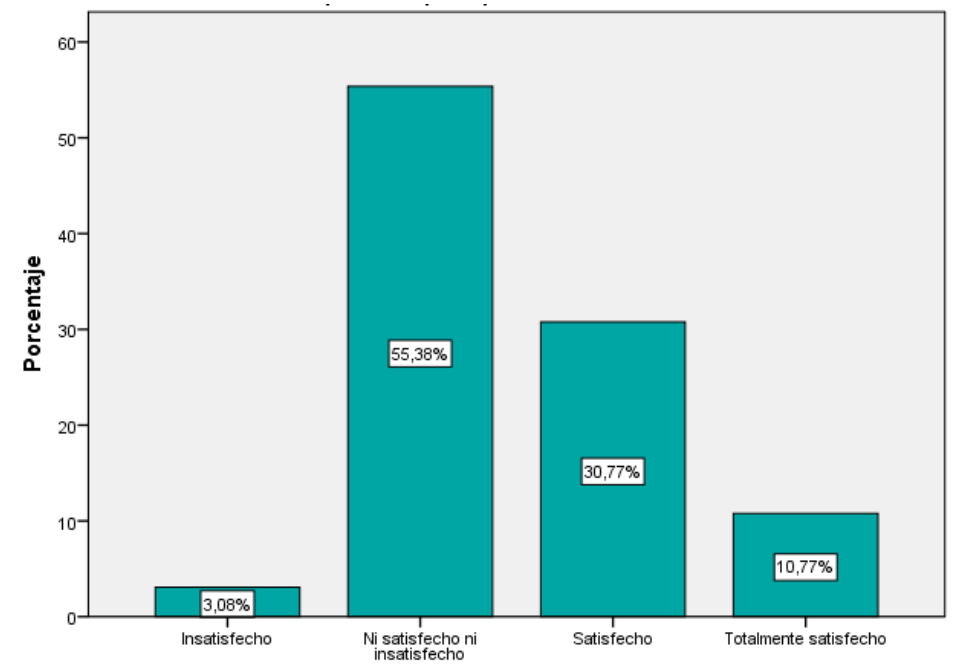

Figura 9 Esperó mucho al personal auxiliar de enfermería de la Fundación Cottolengo para ser atendido 
El 55.38\% de los usuarios encuestados que estuvo ingresado en el área de hospitalización de la Fundación Cottolengo estuvo ni satisfecho ni insatisfecho por el tiempo de espera por ser atendido, el $30.77 \%$ estuvo satisfecho, el $10.77 \%$ totalmente satisfecho y un 3.08\% insatisfecho.

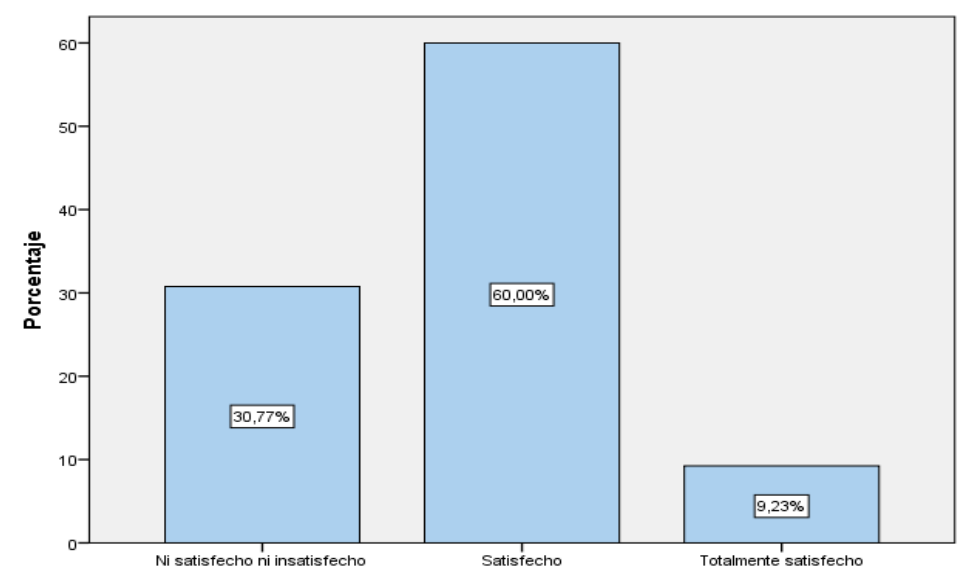

Figura 10 Qué tan amable fue el personal de auxiliar de enfermería al momento de prestar sus servicios en el área de hospitalización de la Fundación Cottolengo

El $60.0 \%$ de los usuarios encuestados que estuvieron ingresados en el área de hospitalización de la Fundación Cottolengo fue satisfecho con la amabilidad que fue atendido por el personal de auxiliar de enfermería, el $30.77 \%$ ni satisfecho ni insatisfecho y el 9.23\% fue totalmente satisfecho.

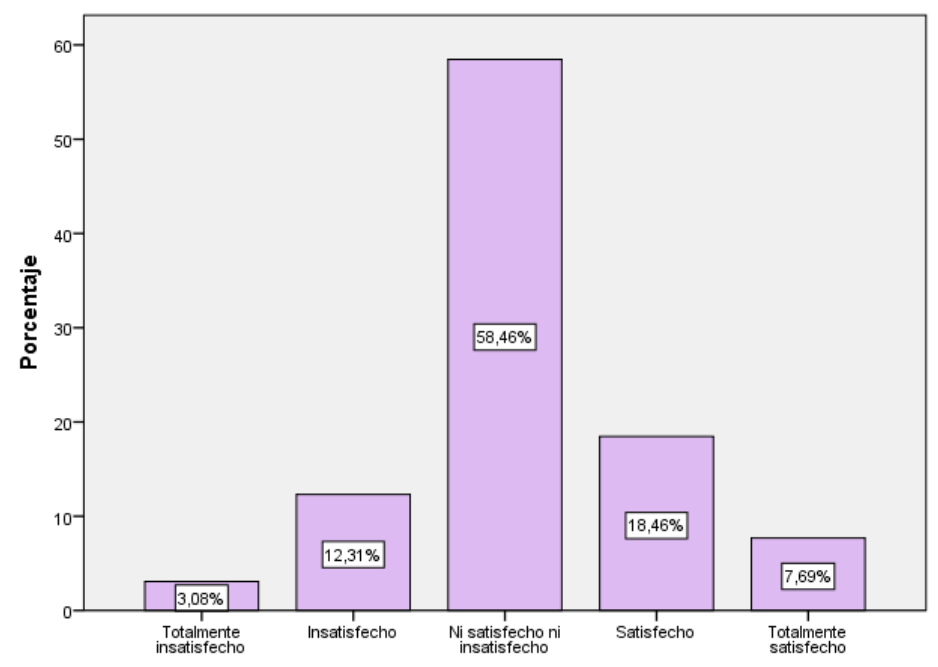

Figura 11. Qué tan dispuesto estuvo el personal de auxiliar de enfermería del área de hospitalización de la Fundación Cottolengo 
El 58.46\% de los usuarios encuestados estuvo ni satisfecho ni insatisfecho con la disponibilidad para ayudar del personal de auxiliar de enfermería del área de hospitalización de la Fundación Cottolengo, el $18.46 \%$ estuvo satisfecho, el $12.31 \%$ insatisfecho, el $7.69 \%$ totalmente satisfecho y un $3.08 \%$ totalmente insatisfecho.

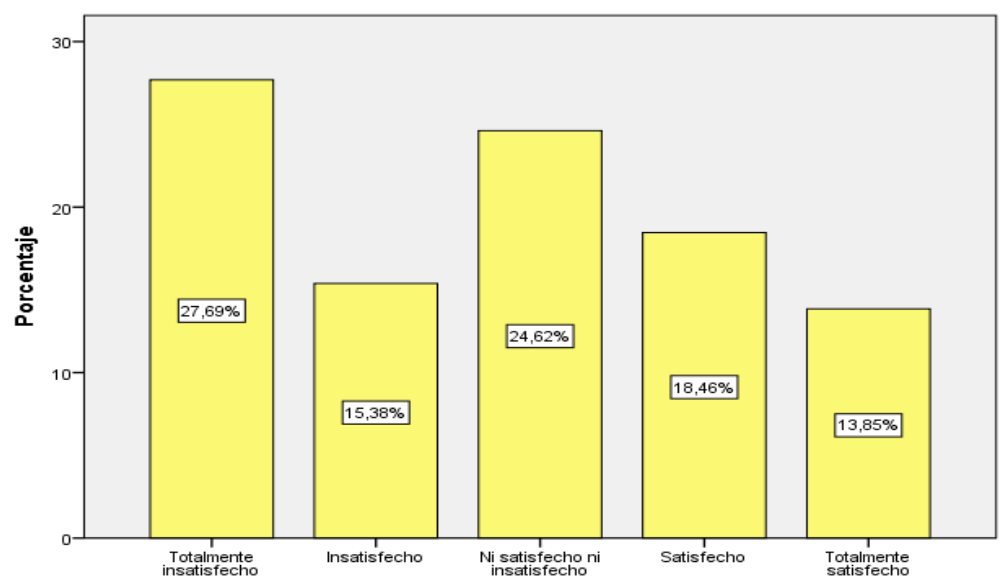

Figura 12 El personal de auxiliar de enfermería fue rápida para conseguir lo que necesitaba cuando estuvo ingresado en el área de hospitalización de la Fundación Cottolengo

El 27.69\% de los usuarios encuestados que estuvieron ingresados en el área de hospitalización de la Fundación Cottolengo fue totalmente insatisfecho por la rapidez de conseguir lo que necesitaba, el $24.62 \%$ estuvo ni satisfecho ni insatisfecho, el $18.46 \%$ estuvo satisfecho, el $15.38 \%$ insatisfecho y el 13.85 totalmente satisfecho.

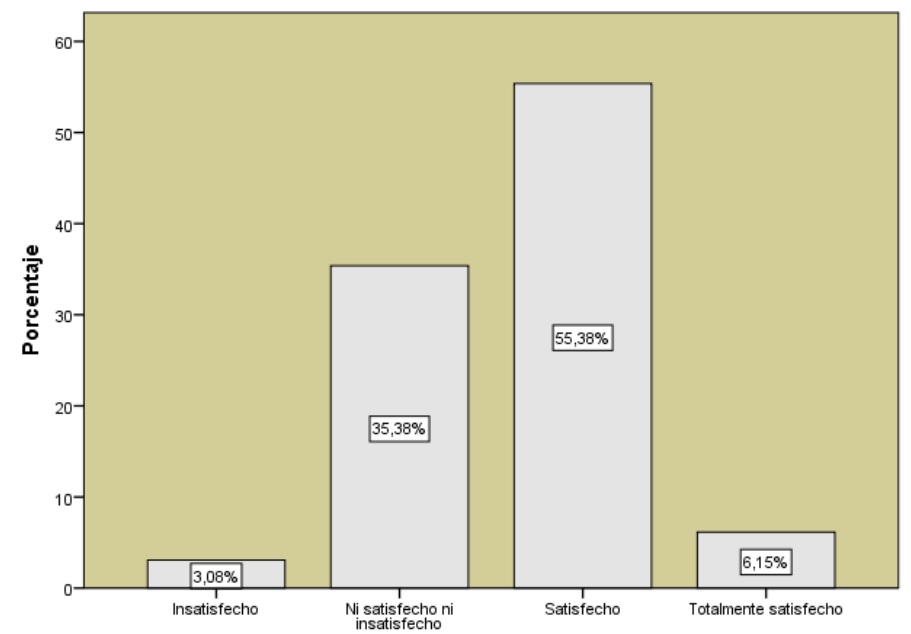

Figura 13 El personal de auxiliar de enfermería estuvo preparado para hacer bien su trabajo en el área de hospitalización de la Fundación Cottolengo 
El 55.38\% de los encuestados que estuvo ingresado en el área de hospitalización de la Fundación Cottolengo fue satisfecho al realizar bien su trabajo, el 35.38\% estuvo ni satisfecho ni insatisfecho, el $6.15 \%$ totalmente satisfecho y el $3.08 \%$ insatisfecho.

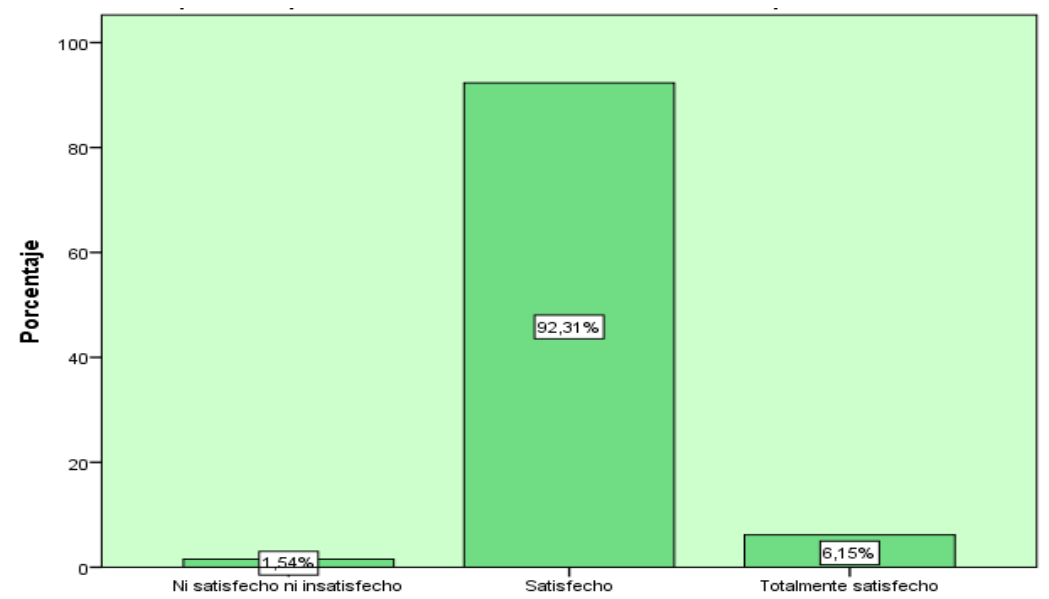

Figura 14 El personal de auxiliar de enfermería tuvo la capacidad de atender las necesidades de los pacientes que estuvieron ingresados en el área de hospitalización de la Fundación Cottolengo

El 92.31\% de los usuarios encuestados que estuvieron ingresados en el área de hospitalización de la Fundación Cottolengo fue satisfecho al atender sus necesidades, el 6.15\% totalmente satisfecho y el $1.54 \%$ ni satisfecho ni insatisfecho

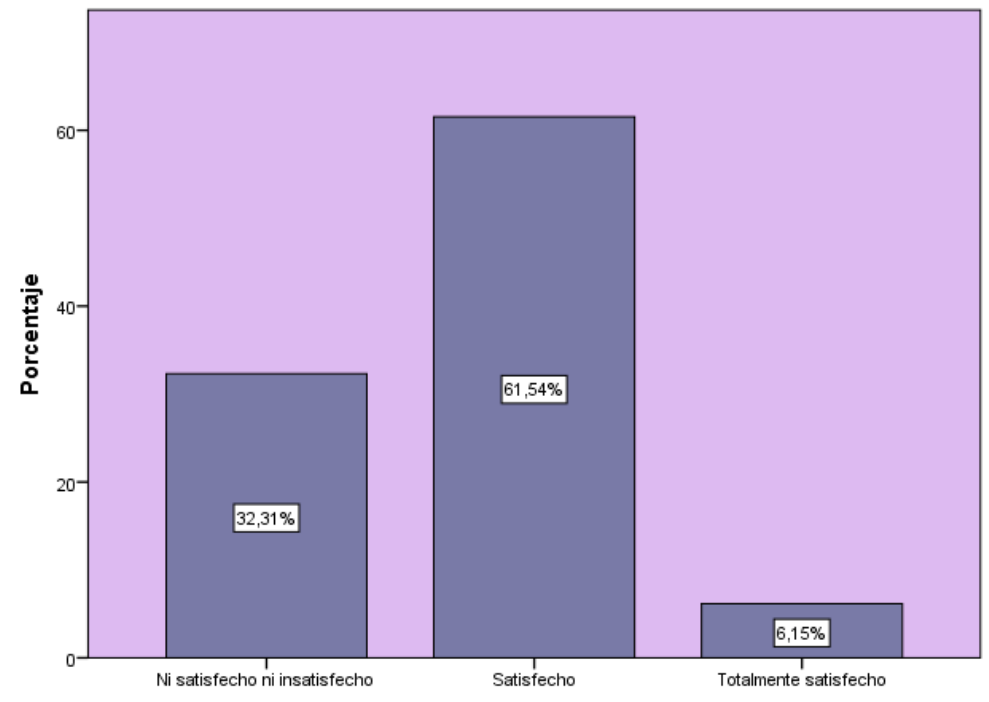

Figura 15 El personal de auxiliar de enfermería tuvo un trato personalizado cuando estuvo en el área de hospitalización de la Fundación Cottolengo 
El 61.54\% de los usuarios encuestados que estuvieron ingresados en el área de hospitalización de la Fundación Cottolengo fue de satisfecho en el trato personalizado del personal de auxiliar de enfermería, el $32.31 \%$ fue ni satisfecho ni insatisfecho, el 6.15\% totalmente satisfecho.

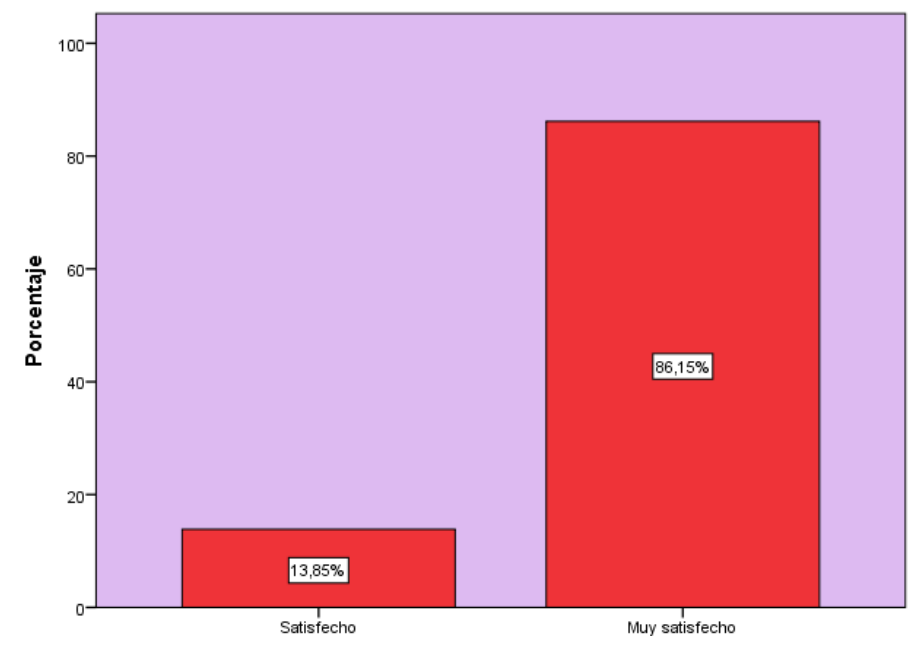

Figura 16 Que le pareció en su totalidad la Fundación Cottolengo

El 86.15\% de los usuarios encuestados que estuvieron ingresados en el área de hospitalización de la Fundación Cottolengo fue de muy satisfecho en su totalidad y un $13.85 \%$ solo satisfecho.

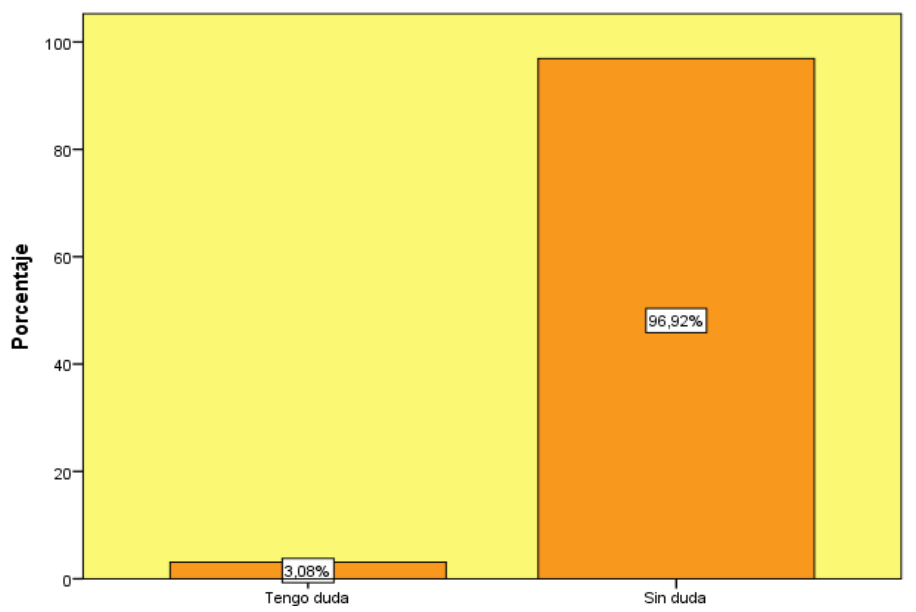

Figura 17 Recomendaría usted los servicios que brinda la Fundación Cottolengo para algún familiar suyo o amigo

El 96.92\% de los usuarios encuestados que estuvieron ingresados en el área de hospitalización de la Fundación Cottolengo sin duda recomendaría los servicios que brinda, el 3.08\% tiene duda en recomendar. 


\section{Análisis de Fiabilidad}

En la tabla 3 muestra el alfa de Cronbach de los constructos de la calidad de los servicios de enfermería. La validación global fue de 0.887 por lo tanto, el instrumento es altamente fiable.

Tabla 3 Medidas de Confiabilidad del constructo

\begin{tabular}{cc}
\hline Alfa de Cronbach & N de elementos \\
\hline 0.887 & 16 \\
\hline
\end{tabular}

Fuente:

Medida Kaiser-Meyer-Olkin de adecuación de muestreo 0.758 y la prueba de esfericidad de Bartlett significativa.

\section{Análisis de Componentes Principales}

En la tabla 4 se presenta la matriz de la varianza total explicada en $74.76 \%$ con cuatro componentes

Tabla 4 Varianza total explicada

\begin{tabular}{c|ccc}
\hline \multirow{2}{*}{ Componente } & \multicolumn{3}{|c}{ Sumas de rotación de cargas al cuadrado } \\
\cline { 2 - 4 } & Total & \% de varianza & \% acumulado \\
\hline 1 & 4,360 & 27,250 & 27,250 \\
2 & 3,517 & 21,981 & 49,231 \\
3 & 2,965 & 18,532 & 67,762 \\
4 & 1,119 & 6,996 & 74,759 \\
\hline
\end{tabular}

Fuente: 
Tabla 5 Matriz de componente rotado

\begin{tabular}{|c|c|c|c|c|}
\hline & & Comp & onente & \\
\hline & 1 & 2 & 3 & 4 \\
\hline Tecnología Biomédica &,- 088 & 195 & ,893 &,- 034 \\
\hline Aspecto del personal & 679 &, 507 &,- 017 &,- 108 \\
\hline Señalización intrahospitalaria & ,232 &, 823 & ,353 &,- 024 \\
\hline Comodidad de las habitaciones &, 115 & ,806 & ,305 & ,002 \\
\hline Interés de las enfermeras & ,636 & ,396 & ,256 & 071 \\
\hline Información que proporcionan & ,326 &, 560 &,- 517 & ,225 \\
\hline Tiempo de espera para ser atendido & ,442 & ,778 &, 021 & ,096 \\
\hline Interés para solucionar problemas &, 525 & ,665 & ,072 &,- 073 \\
\hline Puntualidad & 649 & ,376 & ,247 & ,071 \\
\hline Rapidez para conseguir lo que necesita & ,784 & ,123 & ,099 & 064 \\
\hline Disposición para ayudar &, 860 & ,189 &,- 176 &,- 041 \\
\hline Confianza que transmiten & ,779 &, 145 &,- 174 &,- 224 \\
\hline Amabilidad & 619 & ,099 & ,620 & 084 \\
\hline Preparación para hacer bien el trabajo & ,206 &, 120 & ,842 & 141 \\
\hline Trato personalizado &,- 149 & ,371 & 621 &,- 369 \\
\hline Capacidad para entender las necesidades de los pacientes &,- 103 & 058 &,- 006 & ,901 \\
\hline
\end{tabular}

Método de extracción: análisis de componentes principales.

Método de rotación: Varimax con normalización Kaiser. ${ }^{\mathrm{a}}$

a. La rotación ha convergido en 7 iteraciones. 


\section{Correlación entre las dimensiones}

Las principales correlaciones entre las variables de estudio.

Tabla 7 Matriz de correlaciones

\begin{tabular}{|c|c|c|c|c|c|c|}
\hline & & $\begin{array}{l}\text { Confianza } \\
\text { que } \\
\text { transmiten }\end{array}$ & Amabilidad & $\begin{array}{c}\text { Preparación } \\
\text { para hacer } \\
\text { bien el } \\
\text { trabajo }\end{array}$ & $\begin{array}{c}\text { Trato } \\
\text { personalizado }\end{array}$ & $\begin{array}{c}\text { Capacidad para } \\
\text { entender las } \\
\text { necesidades de } \\
\text { los pacientes }\end{array}$ \\
\hline \multirow[t]{10}{*}{ Correlación } & $\begin{array}{l}\text { Tecnología } \\
\text { Biomédica }\end{array}$ &,- 176 &, 427 &, 711 &, 572 &,- 024 \\
\hline & $\begin{array}{l}\text { Aspecto del personal } \\
\text { Señalización } \\
\text { intrahospitalaria }\end{array}$ & $\begin{array}{l}, 638 \\
, 274\end{array}$ & $\begin{array}{l}, 389 \\
, 447\end{array}$ & $\begin{array}{l}, 122 \\
, 429\end{array}$ & $\begin{array}{l}, 078 \\
, 509\end{array}$ & $\begin{array}{l}-, 063 \\
, 019\end{array}$ \\
\hline & $\begin{array}{c}\text { Comodidad de las } \\
\text { habitaciones }\end{array}$ &, 167 & ,392 & ,417 & ,381 &,- 014 \\
\hline & $\begin{array}{c}\text { Interés de las } \\
\text { enfermeras }\end{array}$ & ,404 & ,495 &, 325 &, 136 &,- 002 \\
\hline & $\begin{array}{l}\text { Información que } \\
\text { proporcionan }\end{array}$ & ,394 &, 032 &,- 145 &,- 274 &, 092 \\
\hline & $\begin{array}{l}\text { Tiempo de espera } \\
\text { para ser atendido }\end{array}$ & ,388 & ,388 & ,241 &, 165 &, 041 \\
\hline & $\begin{array}{c}\text { Interés para } \\
\text { solucionar problemas }\end{array}$ & ,447 &, 450 & , 188 & ,246 &,- 042 \\
\hline & Puntualidad & ,441 & ,499 & ,424 &, 212 &,- 014 \\
\hline & $\begin{array}{c}\text { Rapidez para } \\
\text { conseguir lo que } \\
\text { necesita }\end{array}$ &, 551 &, 568 &, 178 &,- 004 &,- 011 \\
\hline & $\begin{array}{c}\text { Disposición para } \\
\text { ayudar }\end{array}$ & ,712 & ,433 &, 044 &,- 110 &,- 097 \\
\hline
\end{tabular}

Pol. Con. (Edición núm. 33) Vol. 4, No 5, mayo 2019, pp. 204-247, ISSN: 2550 - 682X 


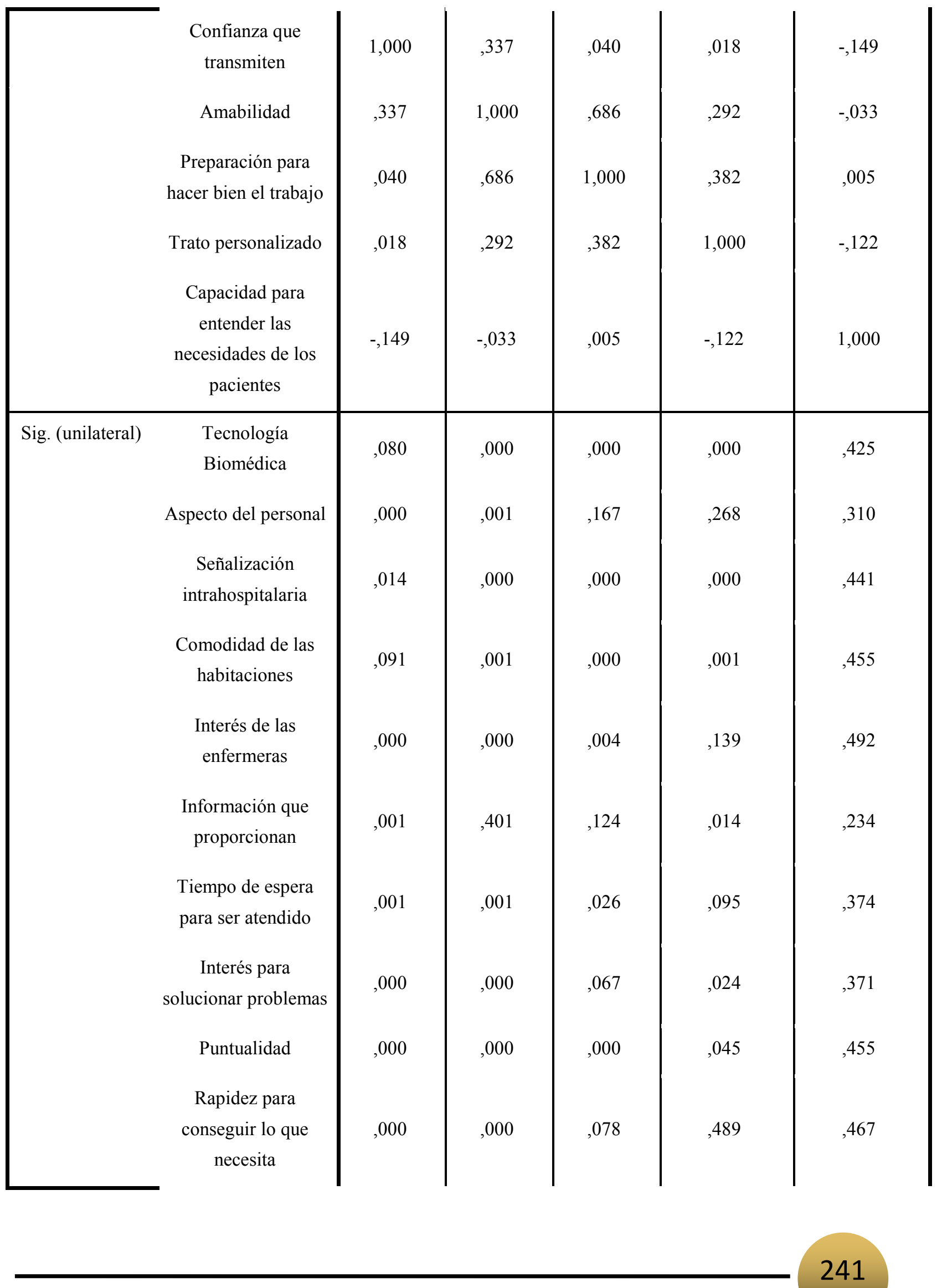




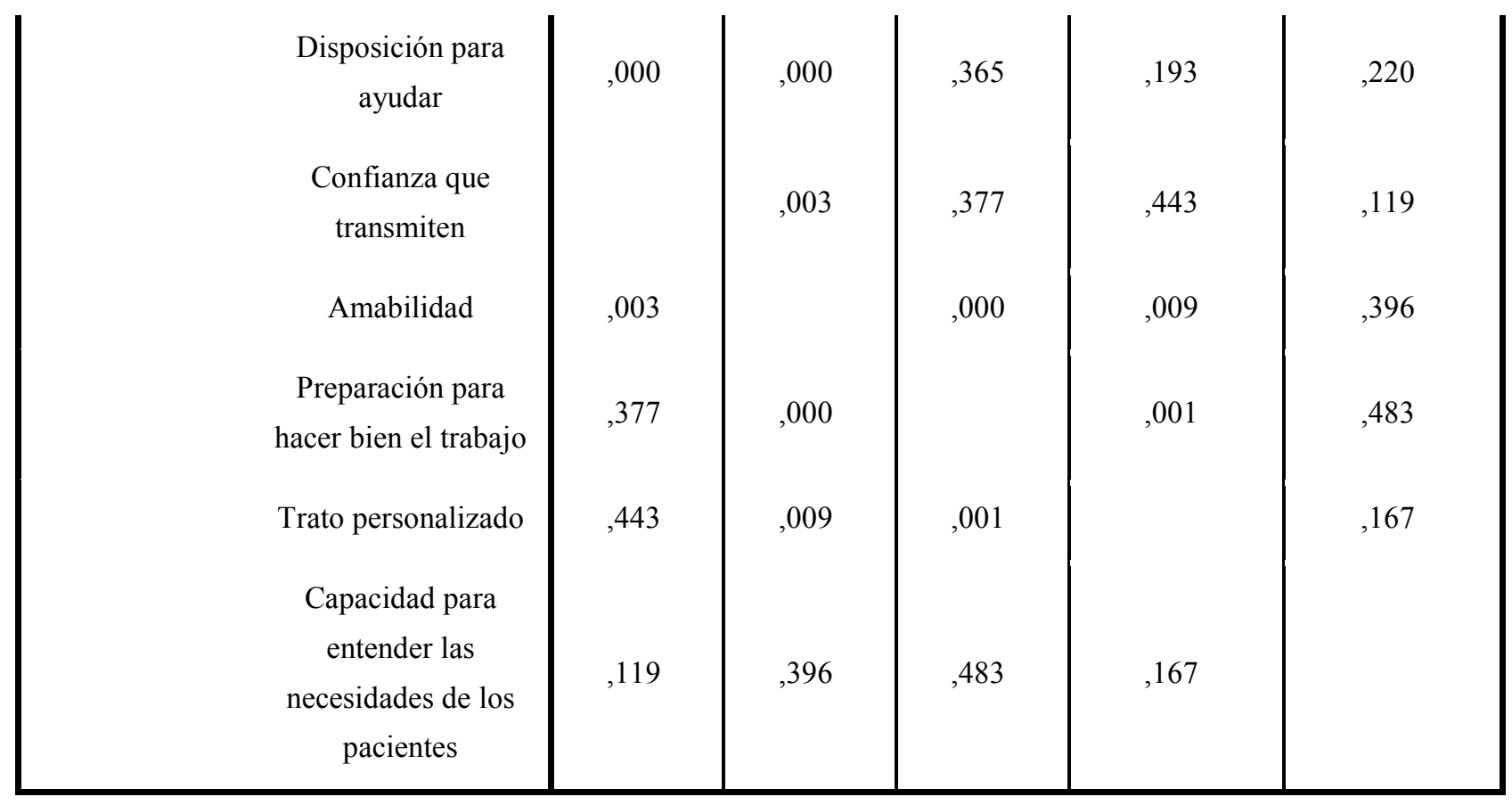

Método de extracción: análisis de componentes principales.

\section{Conclusiones}

En el presente estudio de investigación se encontró que en el Análisis de la Calidad del Servicio de Enfermería por los pacientes ingresados en el área de hospitalización en la Fundación Cottolengo de la ciudad de Manta, fue buena, dicha percepción está relacionada con el nivel de satisfacción global que tienen los pacientes atendidos. Se encontró un nivel de Satisfacción Global de 86.15\% y un porcentaje de Recomendación de la Fundación del 96.92\%, lo cual, según la teoría de desconfirmación de expectativas de Oliver, indica altos niveles de calidad percibida en la Fundación.

Es muy importante señalar que el SERVQHOS original, diseñado por Mira y Aranaz, es un instrumento que ha sido utilizado en más de cinco investigaciones sobre Calidad Percibida, mientras que el SERVQHOS-E, es un cuestionario validado recientemente en el vecino país del norte Colombia para medir Calidad percibida de la atención de Enfermería, por lo tanto, los hallazgos del presente estudio permiten mostrar la eficacia del mismo en la medición del fenómeno, 
pero también permite identificar los elementos que son más valorados de la calidad del Servicio de Enfermería desde el punto de vista del usuario que estuvo ingresado en el área de hospitalización de la Fundación Cottolengo, es decir, desde la percepción que cada uno de los pacientes en un tiempo determinado.

En la revisión de esta investigación se evidenció que si bien existen estudios similares a nivel internacional, éstos han utilizado instrumentos diferentes que miden la satisfacción y la calidad percibida, así como sus escalas de medición y número de ítems que precisan los aspectos específicos al interior del constructo. Por lo antes mencionado no se puede comparar este tipo de resultados en el presente estudio.

Es hasta el momento es el primer estudio en el Ecuador, que se realiza utilizando el instrumento, SERVQHOS-E para medir Calidad del Servicio de Enfermería después de haber sido validado en el vecino país del norte, lo cual obligó al investigador a crear una base de datos para el procesamiento de la información, la cual se elaboró en el programa SPSS versión 24.

Los resultados encontrados en el presente estudio de investigación se entregó en primera instancia a la Universidad para su respectiva validación, y posterior a la Fundación Cottolengo, para establecer una plan de mejoras, en aspectos relacionados con la atención de Enfermería y que son susceptibles de ser percibidos por los pacientes, familiares y amigos que fueron atendidos en el área de hospitalización.

\section{Recomendaciones}

Es recomendable evaluar sistemáticamente la Calidad del Servicio de Enfermería con base en la percepción de los pacientes y analizar la tendencia de los resultados, con el fin de revisar, fortalecer y/o mejorar los factores que determinan la calidad de la atención en la Fundación.

Es importante resaltar que el instrumento SERVQHOS-E mide calidad percibida por parte de los pacientes, por lo tanto, se deberá complementar con otros instrumentos idóneos a fin de acaparar toda la información que debe tenerse para avalar además la calidad técnico científica planteada por Donabedian, así también como otras medidas entre las que se sugieren los propios directivos de la Fundación. 
Se recomienda que el centro especializado en cuidados paliativos establezca una cultura de investigación en todos los procesos internos que permitan el mejoramiento continuo de la calidad, teniendo en cuenta su participación como centro de tercer nivel seguro para el ámbito nacional, ya que se cuenta con la red de salud integral dispuesta por el Ministerio de Salud Pública.

Se recomienda concienciar al personal de Enfermería en aspectos de interrelación terapéutica, con el propósito de mejorar la comunicación y coordinación, para ofrecer a los usuarios una mejor atención; seguir realizando estudios comparativos con otros centros especializados de tercer nivel públicos o privados de la ciudad o el país para poder determinar los elementos que inciden positiva o negativamente en la calidad del Servicio de Enfermería.

Seguir utilizando el Instrumento SERVQHOS-E para evaluar la calidad del Servicio de Enfermería percibida por el paciente y con esto seguir comprobando la confiabilidad del instrumento, no obstante, se recomienda previa validez en el contexto teniendo en cuenta las diferentes culturas; hay que tener en cuenta que el instrumento SERVQHOS-E solo se ha utilizado en instituciones públicas a nivel internacional, se recomienda utilizarlo en Instituciones privadas de salud para comprobar y/o correlacionar resultados, teniendo en cuenta las características de los pacientes atendidos, hospitalizados y el sistema de organización de los servicios de Enfermería.

\section{Referencias Bibliográficas}

ACONA, D 2015 Metodología Cuantitativa. Estrategias y Técnicas de Investigación Social. Metodología Cuantitativa. Estrategias Y Técnicas de Investigación Social.

Álvarez, Francisco 2015. Calidad y Auditoría en Salud. ECOE ediciones.

Barragán, Julián, and Fred Manrique-Abril 2010. Validez E Confiabilidade Do SERVQHOS Para Enfermagem Em Boyacá, Colombia. Avances En Enfermería 28(2): 48-61.

Barragan, Julián, and Claudia Moreno 2012. Calidad percibida por usuarios de enfermería en tres $\begin{array}{llll}\text { hospitales públicos. Gnfermería } & \text { Global }\end{array}$ http://revistas.um.es/eglobal/article/view/153391, accessed September 26, 2017. 
Barragán, Julián, Yeni Pedraza, and Magda Rincón 2013. Perceived Quality of Nursing Care Hospital by Users, Tunja 2011. Revista Facultad Nacional de Salud Pública 31(2): 187193.

Borré, Yeis 2013. Calidad Percibida de La Atención de Enfermería Por Pacientes Hospitalizados En Institución Prestadora de Servicios de Salud de Barranquilla. Universidad Nacional de Colombia.

Carman, James 1988. Consumer Perceptions Of Service Quality: An Assessment Of T - ProQuest. https://search.proquest.com/openview/9b58a6fed9a03970daaa0c1557ae7bb2/1?pqorigsite $=$ gscholar \&cbl=41988, accessed September 28, 2017.

Castro, Laura, Mauricio Rincón, and Dustin Gómez 2017. Health Education: A View from Anthropology. Revista Ciencias de La Salud 15(1): 145-163.

Cogollo-Milanes, Zuleima, Luz Cantillo-Pérez, Laura García-Gómez, Lorena García-Angulo, and Carlos Severiche-Sierra 2017. Calidad de vida relacionada con salud en guardas de seguridad de Cartagena, Colombia. Ciencia y Salud Virtual 8(2): 54-61.

Constituyente, Ecuador Asamblea 2008. Constitución de La República Del Ecuador.

Cook, Colleen, Bruce Thompson, Fred Heath, and Russel Thompson 2001. LibQUAL+: Service Quality Assessment in Research Libraries. IFLA Journal 27(4): 264-268.

Coronado, Roberto, Eva Cruz, Salvador Macías, Aurelia Arellano, and Tania Nava 2013. El contexto actual de la calidad en salud y sus indicadores. Revista Mexicana de Medicina Física y Rehabilitación 25(1): 26-33.

Cronin, Joseph, and Steven Taylor 1992. Measuring Service Quality: A Reexamination and Extension. The Journal of Marketing: 55-68.

Deming, Edwards 1989. Calidad, Productividad Y Competitividad: La Salida de La Crisis. Ediciones Díaz de Santos.

Donabedian, Avedis 1966. Evaluating the Quality of Medical Care. The Milbank Memorial Fund Quarterly 44(3): 166-206. 
1984 La Calidad de La Atención Médica. Prensa Médica Mexicana.

Fundación Cottolengo N.d. Historia, Fundación Cottolengo. http://www.fundacioncottolengo.net/historia/, accessed March 13, 2018.

Galván, Héctor, José Moctezuma, Germán Dolci, and David López 2016. De la idea al concepto en la calidad en los servicios de salud. Revista CONAMED 17(4).

Hernández, Luis 2012. Metodología de la investigación en ciencias de la salud. Ecoe Ediciones.

Hernández, Roberto, Carlos Fernández-Collado, and Pilar Baptista 1998. Metodología de La Investigación, vol.1. 4ta edition. Mcgraw-hill México.

INEC N.d. Manabí Resumen Censo Población Y Vivienda 2010. Scribd. https://es.scribd.com/doc/100658998/Manabi-resumen-Censo-Poblacion-y-Vivienda2010, accessed March 2, 2018.

ISO 9000: 2005, Norma 2000 9000: 2005 "Sistemas de Gestión de La Calidad. Fundamentos Y Vocabulario.” AENOR, Madrid: 8-10.

Lankshear, Colin, and Michele Knobel 2000. Problemas Asociados Con La Metodología de La Investigación Cualitativa. Perfiles Educativos 22(87): 6-27.

Losada, Mauricio, and Augusto Rodríguez 2007. Calidad Del Servicio de Salud: Una Revisión a La Literatura Desde La Perspectiva Del Marketing. Cuadernos de Administración 20(34): $237-258$.

McDougall, Gordon, and Terrence Levesque 1995 A Revised View of Service Quality Dimensions. Journal of Professional Services Marketing 11(1): 189-210.

Ministerio de Coordinación de la Política y Gobiernos Autónomos Descentralizados 2012. Código Orgánico de Organización Territorial, Autonomía Y Descentralización. COOTAD. Quito, Ecuador.

Mira, Jose, Jesús Aranaz, Jesús Rodríguez-Marín, et al. 1998. SERVQHOS: Un Cuestionario Para Evaluar La Calidad Percibida de La Asistencia Hospitalaria, vol.4. 
Mira Solves, Aina Buil, Rodríguez-Marín, and Andrés Aranaz 1997. Calidad Percibida Del Cuidado Hospitalario. Gaceta Sanitaria 11(4): 176-189.

Monar-Merchán, Carlos, and Geovanny Arauz-Barcia 2016. La Política de Desarrollo En El Área Productiva, Provincia de Manabí. Ecuador. Dominio de Las Ciencias 2(3): 211-225.

Oliver, Richard 1993 A Conceptual Model of Service Quality and Service Satisfaction: Comparative Goals, Different Concepts. Advances in Service Marketing and Management 2: $65-85$.

OMS 1946 Constitución de La Organización Mundial de La Salud. Génova, Italia.[Fecha de Ingreso 13 de Enero de 2015). Disponible En La Web: Http://wvvw. Who. Int/governace/eb/whoconstitudon_sp. Pdf.

Oviedo, H. C., and A. Campo-Arias 2014 Aproximación Al Uso Del Coeficiente Alfa de Cronbach. Rev Col Psiqui 2005; 36 (4): 572-580. Acceso: 06-19.

Parasuraman, Ananthanarayanan, Valarie Zeithaml, and Leonard Berry 1988 Servqual: A Multiple-Item Scale for Measuring Consumer Perc. Journal of Retailing 64(1): 12.

Reyes, Eva 2015. Fundamentos de enfermería: Ciencia, metodología y tecnología. Editorial El Manual Moderno.

Rubio, Elby, Rita Navas, Ada Chacín, Jairelis Bracho, and Kelly González 2016 Salud Bucal En Las Aulas Hospitalarias: Una Propuesta Educativa Para El Servicio de Oncología. Ciencia Odontológica 13(2): 75-89.

Saleh, Farouk, and Chris Ryan 1991 Analysing Service Quality in the Hospitality Industry Using the SERVQUAL Model. Service Industries Journal 11(3): 324-345.

Sarmadi, Mohammad 2014 Florence Nightingale Who Raised Nursing as a Highly Profession. International Journal of Nursing Science 4(3): 33-36.

Swan, J., M. Carroll, and I. Bloomington 1980 Patient Satisfaction and Overview of Research-1965 to 1978: Refining Concepts and Measures of Consumer Satisfaction and Complaining Behaviour. Indiana. Indiana University. 